\title{
Buoyant-thermocapillary instabilities in extended liquid layers subjected to a horizontal temperature gradient
}

\author{
J. Burguete ${ }^{a)}$ \\ Service de Physique l'État Condensé, CEA/Saclay, F-91191 Gif-sur-Yvette, France \\ and Departamento de Física y Matemática Aplicada, Universidad de Navarra, E-31080 Pamplona, Spain \\ N. Mukolobwiez, F. Daviaud, N. Garnier, and A. Chiffaudel ${ }^{\text {b) }}$ \\ Service de Physique l'État Condensé, CEA/Saclay, F-91191 Gif-sur-Yvette, France
}

(Received 11 May 2000; accepted 8 June 2001)

\begin{abstract}
We report experiments on buoyant-thermocapillary instabilities in differentially heated liquid layers. The results are obtained for a fluid of Prandtl number 10 in a rectangular geometry with different aspect ratios. Depending on the height of liquid and on the aspect ratios, the two-dimensional basic flow destabilizes into oblique traveling waves or longitudinal stationary rolls, respectively, for small and large fluid heights. Temperature measurements and space-time recordings reveal the waves to correspond to the hydrothermal waves predicted by the linear stability analysis of Smith and Davis [J. Fluid Mech. 132, 119 (1983)]. Moreover, the transition between traveling and stationary modes agrees with the work by Mercier and Normand [Phys. Fluids 8, 1433 (1996)] even if the exact characteristics of longitudinal rolls differ from theoretical predictions. A discussion about the relevant nondimensional parameters is included. In the stability domain of the waves, two types of sources have been evidenced. For larger heights, the source is a line and generally evolves towards one end of the container leaving a single wave whereas for smaller heights, the source looks like a point and emits a circular wave which becomes almost planar farther from the source in both directions. () 2001 American Institute of Physics. [DOI: 10.1063/1.1398536]
\end{abstract}

\section{INTRODUCTION}

In the past few decades, there has been a growing interest for buoyant-thermocapillary flows both on fundamental and applied aspects. First, there is the need for understanding and controlling the instabilities of these flows in many applications such as floating zone crystal growth, ${ }^{1}$ electron beam vaporization or laser welding. In particular, experiments are being carried out to explore the possibility of manufacturing crystals in space, where gravity is negligible and only surface tension forces are relevant. As the dynamics observed in these applications is very complex because of the presence of many parameters, it is worth working in a simple configuration with well-controlled experimental conditions, in order to be closer to the theoretical models. Second, theses flows provide very interesting systems of traveling waves which can be described by envelope equations such as the complex Ginzburg-Landau equation. ${ }^{2}$ These wave-systems allow a careful study of the transition to spatio-temporal chaos.

Our study concerns a buoyant-thermocapillary flow in a rectangular container of dimensions $\left(L_{x}, L_{y}\right)$ filled with a Boussinesq fluid layer of depth $h$, with a free surface and subjected to a horizontal temperature difference $\Delta T$ along $x$ axis. This system is characterized by a set of dimensionless parameters:

(i) Marangoni number $M a(\beta, d)=\gamma \beta d^{2} / \rho \kappa \nu$;

(ii) Rayleigh number $\operatorname{Ra}(\beta, d)=g \alpha \beta d^{4} / \kappa \nu$;

${ }^{a)}$ Electronic mail: javier.burguete@ fisica.unav.es

${ }^{b)}$ Also at: Centre National de la Recherche Scientifique. (iii) Prandtl number $P=\nu / \kappa$;

(iv) Dynamic Bond number $B o=R a / M a$;

(v) Capillary number $C a=\gamma \Delta T / \sigma$;

(vi) Aspect ratios $\Gamma_{x}=L_{x} / h, \Gamma_{y}=L_{y} / h$, and $\Gamma=L_{y} / L_{x}$,

where $g$ is the gravitational acceleration, $\alpha$ the thermal expansion coefficient, $\kappa$ the thermal diffusivity, $\rho$ the density of the fluid, $\nu$ the kinematic viscosity, $\sigma$ the surface tension and $\gamma=-(\partial \sigma / \partial T) . \beta$ stands for a temperature gradient and $d$ for a typical length: They will be specified when necessary.

Smith and Davis ${ }^{3,4}$ (SD) performed a linear stability analysis of an infinite fluid layer with a free surface, without gravity nor heat exchange to the atmosphere. They found two types of instabilities: Stationary longitudinal rolls and oblique hydrothermal waves depending on the Prandtl number and on the basic flow considered (with or without return-flow profile). The mechanisms for the hydrothermal wave instability have been described by Smith. ${ }^{5}$

The stability of buoyant-thermocapillary instabilities has been addressed by Laure and Roux ${ }^{6}$ for low Prandtl numbers and by Gershuni et al. ${ }^{7}$ and Parmentier et al. ${ }^{8}$ for values of the Prandtl number up to 10 . The former analysis considers the case of adiabatic surfaces whereas the latter considers conducting boundary conditions. A recent study has been performed by Normand and Mercier ${ }^{9}$ including gravity and a Biot number introduced to describe the heat transfer at the top free surface. In a conductive state, the Biot number is defined as $B i=\lambda_{a} h / \lambda_{f} h_{a}$ where $\lambda_{a}$ and $\lambda_{f}$ are, respectively, the thermal conductivity of air and fluid, and $h_{a}$ is the effective height of the air layer. This study shows the existence of 
TABLE I. Different experimental conditions and observations (see Introduction for details). In each group, entries have been ordered by increasing Prandtl number. The first group contains "small" $y$-aspect-ratio experiments (S). The second and third group contain "large" $y$-aspect-ratio experiments $(\mathrm{L})$ that report waves propagating along both $x$ and $y$. HW stands for hydrothermal waves and BLW for boundary-layer waves. In the annular configurations, $L_{x}=R_{o}-R_{i}$ and $L_{y}=\pi\left(R_{o}+R_{i}\right)$, where $R_{o}$ and $R_{i}$ are, respectively, the outer and inner radius of the cell.

\begin{tabular}{lllllll}
\hline \hline \multicolumn{1}{c}{ Experiment } & Geometry & $L_{x}(\mathrm{~mm})$ & $L_{y}(\mathrm{~mm})$ & $h(\mathrm{~mm})$ & $P$ & Obs. \\
\hline Villers and Platten & rectangular & 30 & 10 & $1.75-14.25$ & 4.2 & $\mathrm{~S}$ \\
Braunsfurth and Homsy & rectangular & 10 & 10 & $1.25-10$ & 4.4 & $\mathrm{~S}$ \\
Gillon and Homsy & rectangular & 10 & 38 & 6.8 & 9.5 & $\mathrm{~S}$ \\
Garcimartín et al. & rectangular & 100 & 10 & $2-3.5$ & $10,15,30$ & $\mathrm{~S}$ \\
Kamotani et al. & annular & $2-15$ & $6-47$ & $\sim 5-60$ & 10,25 & $\mathrm{~S}$ \\
De Saedeleer et al. & rectangular & 74 & 10 & $2.5-4.7$ & 15 & $\mathrm{~S}$ \\
Pelacho and Burguete & rectangular & 60 & 50 & $1.25-3.5$ & 10 & L-HW \\
Daviaud and Vince & rectangular & 10 & 200 & $0.6-10$ & 10.3 & L-HW \\
Mukolobwiez et al. & annular & 10 & 503 & 1.7 & 10.3 & L-HW \\
Riley and Neitzel & rectangular & 30 & 50 & $0.75-2.5$ & 14 & L-HW \\
Schwabe et al. & annular & 57 & 305 & $0.5-3.6$ & 17 & L-HW \\
Ezersky et al. & rectangular & 70 & 50 & $1.2-3.1$ & 60 & L-BLW \\
Ezersky et al. & annular & 40 & 188 & $2-8$ & 60 & L-BLW \\
This study & rectangular & $10,20,30$ & $30-250$ & $0.5-10$ & 10.3 & L-HW \\
\hline \hline
\end{tabular}

a transition from oscillatory to stationary modes when the Bond number (the ratio between the Rayleigh and the Marangoni number) is increased. This transition depends on the Biot number.

Priede and Gerbeth ${ }^{10}$ have applied the concepts of convective, absolute, and global instabilities to this problem, to calculate the thresholds of spatial and temporal oscillations of the flow. Finally, a weakly nonlinear stability analysis has been performed by Smith. ${ }^{11}$ In particular, he has determined the possible equilibrium wave forms for the instability above threshold and analyzed their destabilization.

The characteristics of two-dimensional (2D), and more recently three-dimensional (3D), buoyant-thermocapillary driven flows have been investigated by numerical simulations. Carpenter and Homsy ${ }^{12}$ performed simulations for $P$ $=1$ and large $\Delta T$ varying the Bond number and Ben Hadid and Roux ${ }^{13}$ for low-Prandtl number fluids and various values of the aspect ratio. The latter have shown the existence of a multicellular steady state and a transition to oscillatory convection. Villers and Platten ${ }^{14}$ have carried out both experiments and numerical simulations for $P=4$ which confirm the existence of the multicellular flow. In a recent study, Mercier and Normand ${ }^{15}$ provided a general study of the structure of this multicellular flow with respect to the Prandtl number. They showed the recirculation eddies to originate on the hot (resp. cold) side of the cell for high (resp. low) Prandtl number. Mundrane and Zebib ${ }^{16}$ have studied in detail the stability boundaries for the onset of oscillatory convection, for different value of $P$ and the aspect ratio. More recently, $\mathrm{Xu}$ and Zebib $^{17}$ have performed $2 \mathrm{D}$ and $3 \mathrm{D}$ calculations for fluids with Prandtl number between 1 and 10. They have determined the Hopf bifurcation neutral curves as function of the Reynolds number and the aspect ratio and considered the influence of sidewalls.

The existence of thermocapillary instabilities, and in particular of oscillations, has been demonstrated in numerous experiments, but only few of them have provided a direct evidence of hydrothermal waves (see below and Table I). In many experiments, the basic flow was observed to destabilize first against a stationary multicellular instability before exhibiting oscillatory behaviors.

Preisser et al. ${ }^{1}$ and Velten et al. ${ }^{18}$ have shown in floating half zones from molten salts $(P=8$ to 9$)$ that the primary flow becomes unstable towards an oscillatory traveling instability. Villers and Platten ${ }^{14}$ performed careful velocity measurements on a narrow channel $\left(L_{x}=30 \mathrm{~mm}\right.$ and $L_{y}$ $=10 \mathrm{~mm})$ in acetone $(P=4.2)$. They showed the existence of three states: Steady monocellular and multicellular states and oscillatory state. The structure of the oscillating flow is not known but, because $\Gamma_{y}$ is small, one can think that this oscillation does not correspond to hydrothermal waves but to an oscillation of the multicellular pattern. This hypothesis is confirmed by the study of De Saedeleer et al. ${ }^{19}$ performed in a $L_{x}=74 \mathrm{~mm}, L_{y}=10 \mathrm{~mm}$ channel with decane $(P=15)$. They report that before the appearance of waves, stable corotative rolls span over the whole liquid layer, starting from the hot side. A further increase of the temperature gradient leads to a time periodic pattern. Using a $L_{x}=10 \mathrm{~cm}, L_{y}$ $=1 \mathrm{~cm}$ channel filled with fluids with different Prandtl numbers, Garcimartín et al. ${ }^{20}$ suggest that this oscillation is due to a hot boundary layer instability. In the same way, the instabilities obtained by Ezersky et al. in a cylindrical geometry $^{21}$ and a rectangular geometry ${ }^{22}$ for $P \simeq 60$ do not correspond to hydrothermal waves but probably also to an instability of the multicellular flow: The waves propagate from the hot wall towards the cold and do not present the predicted characteristics.

Schwabe et al. ${ }^{23}$ also observed this multicellular flow before waves showed up, but reported the existence of two different oscillatory instabilities in an annular channel $\left(L_{x}\right.$ $=57 \mathrm{~mm}$ and $\left.L_{y} \simeq 305 \mathrm{~mm}\right)$ filled with ethanol $(P=17):$ A short wavelength instability for small heights $(h<1.4 \mathrm{~mm})$ 
with waves traveling in the azimuthal direction and a long wavelength instability for $h>1.4 \mathrm{~mm}$, with larger surface deformations, and waves traveling in the radial and azimuthal directions. ${ }^{24}$ The former seems to be in accordance with the hydrothermal waves predicted by theory, even if some characteristics-e.g., wave number-are not similar, whereas the latter could be related to a surface-wave instability. ${ }^{25}$

Daviaud and Vince $^{26}$ report the existence of traveling waves in a rectangular channel $\left(L_{x}=10 \mathrm{~mm}\right.$ and $L_{y}$ $=200 \mathrm{~mm})$ filled with silicone oil $(P=10)$ and a transition to stationary rolls when the height of liquid is increased. The waves present all the characteristics of hydrothermal waves except for the angle between the wave vector $\boldsymbol{k}$ and the applied horizontal temperature gradient: The propagation is nearly perpendicular to the applied temperature gradient. We will see in the following that this effect is due to relative shortness of the streamwise aspect ratio.

Riley and Neitzel ${ }^{27}$ also observed oblique hydrothermal waves in a rectangular geometry $\left(L_{x}=30 \mathrm{~mm}, L_{y}=50 \mathrm{~mm}\right)$ for silicone oil $(P=14)$ for sufficiently thin layers $(h$ $\leqslant 1.25 \mathrm{~mm}$ ). But for thicker layers, a steady multicellular flow is evidenced which becomes time-dependent when the temperature gradient is increased. Pelacho and Burguete,${ }^{28}$ in a rectangular cell $\left(L_{x}=60 \mathrm{~mm}, L_{y}=50 \mathrm{~mm}\right.$, and $\left.P=10\right)$ observed a multicellular flow and hydrothermal waves. Moreover, they observe a different instability for $h \geqslant 3.5 \mathrm{~mm}$ and large $\Delta T$, which propagates downstream and could be of the same type as the oscillations reported by Villers and Platten, ${ }^{14}$ De Saedeleer et al.,${ }^{19}$ Garcimartín et al. ${ }^{20}$ and Ezersky et al. ${ }^{21,22}$

Kamotani et al. ${ }^{29}$ have performed an experimental study of oscillatory convection in a confined cylindrical container while Gillon and Homsy ${ }^{30}$ have studied combined convection in a confined geometry, in a regime where thermocapillary and buoyancy are of equal importance $\left(h=6.8 \mathrm{~mm}, L_{x}\right.$ $=10 \mathrm{~mm}, L_{y}=38 \mathrm{~mm}, P=9.5$ ). They reported a transition from 2D steady convection to $3 \mathrm{D}$ steady convection, in agreement with the numerical computations of Mundrane and Zebib. ${ }^{16}$ Braunsfurth and Homsy ${ }^{31}$ performed velocity measurements for different heights in a confined container with equal aspect ratios $\left(L_{x}=L_{y} \simeq 1 \mathrm{~cm}, P=4.4\right)$. They found the transition from 2D flow to 3D steady longitudinal rolls to depend on the aspect ratio, in agreement with the experimental results of Daviaud and Vince ${ }^{26}$ and the modelization of Mercier and Normand. ${ }^{9}$ Moreover, they observed an oscillatory flow for higher values of $\Delta T$.

The nonlinear dynamics of these waves has also been studied: Mukolobwiez et $a l .{ }^{32}$ have shown the existence of a supercritical Eckhaus instability in an annular container $\left(L_{x}\right.$ $=10 \mathrm{~mm}, L_{y} \simeq 503 \mathrm{~mm}$, and $\left.P=10\right)$ and Burguete et al. ${ }^{33}$ spatiotemporal defects corresponding to amplitude holes in the rectangular container used in this study.

Finally, let us mention that traveling waves with characteristics similar to hydrothermal waves have been observed in other experimental configurations: hot wire under the surface of a liquid (Vince and Dubois ${ }^{34}$ ) or liquid layer locally heated on its free surface (Favre et al. ${ }^{35}$ ).

In this article, we consider buoyant-thermocapillary instabilities in a rectangular geometry in which the height and
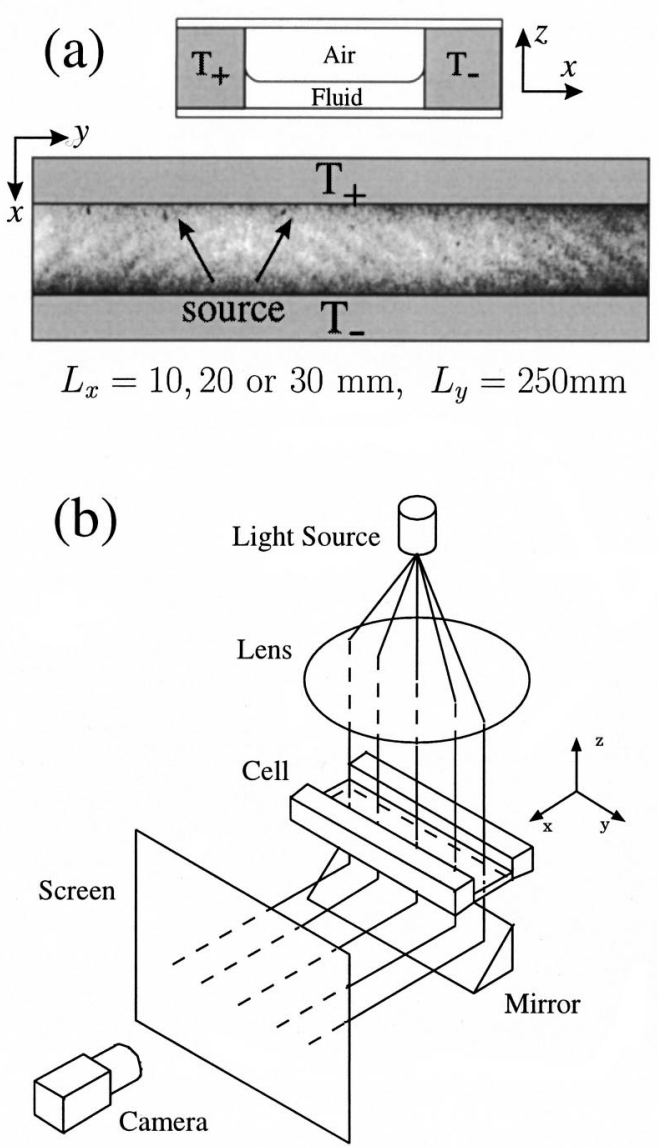

FIG. 1. Schematic drawing of the experimental apparatus. (a) Rectangular container: Cross section (top) and top view (bottom). A liquid layer of depth $h$ is subjected to a horizontal temperature gradient. The origin of coordinates is located on the hot side for $x$ and at the bottom plate for $z$. (b) Optical setup used to perform shadowgraphy.

the aspect ratios have been varied over a large range. In particular, the characteristics of oblique traveling waves and stationary longitudinal rolls have been studied in details and compared to available theories. Special attention has been given to the stability domain of the waves where two kinds of sources of waves have been evidenced.

In the following, we first describe in Sec. II the experimental setup and the measurements techniques. Section III is devoted to the experimental results, concerning the basic flow, the oblique traveling waves, the longitudinal stationary rolls, and finally the destabilization of these patterns further from threshold. The discussion of our results in Sec. IV, concerns the relevance of the scaling parameters and a comparison to theoretical and experimental results. A summary and a conclusion are given in Sec. V.

\section{EXPERIMENTAL SETUP}

The apparatus is an evolution of the one described used by Daviaud and Vince. ${ }^{26}$ It consists of a rectangular container $L_{x}$ long and $L_{y}$ wide as shown in Fig. 1. Most of the experiments have been carried out with $L_{y}=250 \mathrm{~mm}$ and $L_{x}=10,20$, and $30 \mathrm{~mm}$, and in some experiments, $L_{y}$ has been varied down to $30,50,75,90$, and $180 \mathrm{~mm}$. For the 
TABLE II. Physical properties of Rhône Poulenc silicone oil $0.65 \mathrm{cSt}$ at $20^{\circ} \mathrm{C}$. Surface tension measurements have been performed by J. K. Platten at University of Mons-Hainaut (Belgium). Other properties are from the technical data sheet.

\begin{tabular}{ccccccc}
\hline \hline $\begin{array}{c}v \\
\left(\mathrm{~m}^{2} / \mathrm{s}\right)\end{array}$ & $\begin{array}{c}\rho \\
\left(\mathrm{kg} / \mathrm{m}^{3}\right)\end{array}$ & $\begin{array}{c}\alpha \\
\left(\mathrm{K}^{-1}\right)\end{array}$ & $\begin{array}{c}\kappa \\
\left(\mathrm{m}^{2} / \mathrm{s}\right)\end{array}$ & $\begin{array}{c}\sigma \\
(\mathrm{N} / \mathrm{m})\end{array}$ & $\begin{array}{c}\gamma=-\partial \sigma / \partial T \\
(\mathrm{~N} / \mathrm{mK})\end{array}$ & $P$ \\
\hline $6.5 \times 10^{-7}$ & 760 & $1.34 \times 10^{-3}$ & $6.3 \times 10^{-8}$ & $1.59 \times 10^{-2}$ & $8 \times 10^{-5}$ & 10.3 \\
\hline \hline
\end{tabular}

range of fluid depths examined-1 $\mathrm{mm} \leqslant h \leqslant 10 \mathrm{~mm}$ - these dimensions correspond to streamwise (resp. spanwise) aspect ratios $1 \leqslant \Gamma_{x}=L_{x} / h \leqslant 30$ (resp. $\left.3 \leqslant \Gamma_{y}=L_{y} / h \leqslant 250\right)$. The shorter walls are made of Plexiglas, while the two longer vertical walls are made of copper and are thermally regulated by circulating water at different temperatures $T_{+}$and $T_{-}$. The lower boundary consists of a float glass plate and a Plexiglas plate is inserted a few millimeters above the surface of the fluid to reduce evaporation. With this configuration, the height of liquid remains constant to better than $1 \%$ over an experimental run. The same experiment was performed in an annular geometry with a gap between the inner cylinder and the outer cylinder being $10 \mathrm{~mm}$ and the mean perimeter $503 \mathrm{~mm}^{32,36}$ In this last experiment, the main characteristics of the wave patterns are similar to those obtained in the rectangular geometry with $L_{x}=10 \mathrm{~mm}$.

The working fluid used is a $0.65 \mathrm{cSt}$ Rhône-Poulenc silicone oil whose physical properties are given in Table II. This liquid presents a low susceptibility to surface contamination, a medium Prandtl number $P=10$ and is transparent to visible light. Another fluid was used (2 cSt silicone oil, $P=30)$ to check the existence of hydrothermal waves for larger Prandtl numbers.

The fluid depth $h$ is measured with a $0.05 \mathrm{~mm}$ precision and a meniscus is present at the boundaries. The horizontal temperature difference $\Delta T=T_{+}-T_{-}$, is imposed by the two copper walls and is measured using thermocouples. This parameter is regulated with a stability better than $2 \times 10^{-2} \mathrm{~K}$. Room temperature is kept constant near $20^{\circ} \mathrm{C}$ and the hot and cold temperatures are adjusted so that the container mean temperature remains close to it.

The flow is characterized by temperature measurements and visualizations associated to image processing. Temperature measurements are performed using a moving $\mathrm{Cr}-\mathrm{Al}$ (type K) $0.25 \mathrm{~mm}$ thermocouple with a time response small enough to perform temporal temperature series with a $20 \mathrm{~Hz}$ maximal sampling rate. The accuracy of these measurements is typically $0.03 \mathrm{~K}$. Shadowgraphy is used to control the perturbation of the flow due to the presence of the probe. We visualize the streamlines by sowing the flow with Merck Iriodin 100 Silver Pearl and by illuminating it with a $\mathrm{He}-\mathrm{Ne}$ laser light sheet in different vertical planes. The patterns are also visualized by shadowgraphic imaging. A parallel vertical light beam crosses the container from top to bottom and forms a horizontal picture on a screen, due to surface deformations at the oil-air interface and to temperature gradients in the fluid. The surface deformations have been measured, looking at the deflection of a laser beam on the surface of the fluid. These measures give a deformation of less than $15 \mu \mathrm{m}$ in agreement with the value of the capillary number $\mathrm{Ca}$
$=0.005 \mathrm{~K}^{-1} \times \Delta T$. As the surface deflection is small, the shadowgraphy method gives mainly the temperature gradient field variation. ${ }^{37}$

The spatio-temporal evolution of the structures is recorded using a CCD video camera and the images are digitized to 256 gray levels along a line of 512 pixels perpendicular to the gradient. This method is described in detail elsewhere. ${ }^{26}$ Note that the observed region is limited to 185 $\mathrm{mm}$ by the optical setup. Some experiments have been performed in a $L_{y}=180 \mathrm{~mm}$ reduced cell to avoid this limitation. This will be indicated when needed. To extract the pattern behavior from the spatiotemporal diagrams, space and time Fourier transforms and complex demodulation techniques are used. ${ }^{33}$ They allow a determination of the amplitude of the oscillations, the wave number, the frequency and thus the phase velocity.

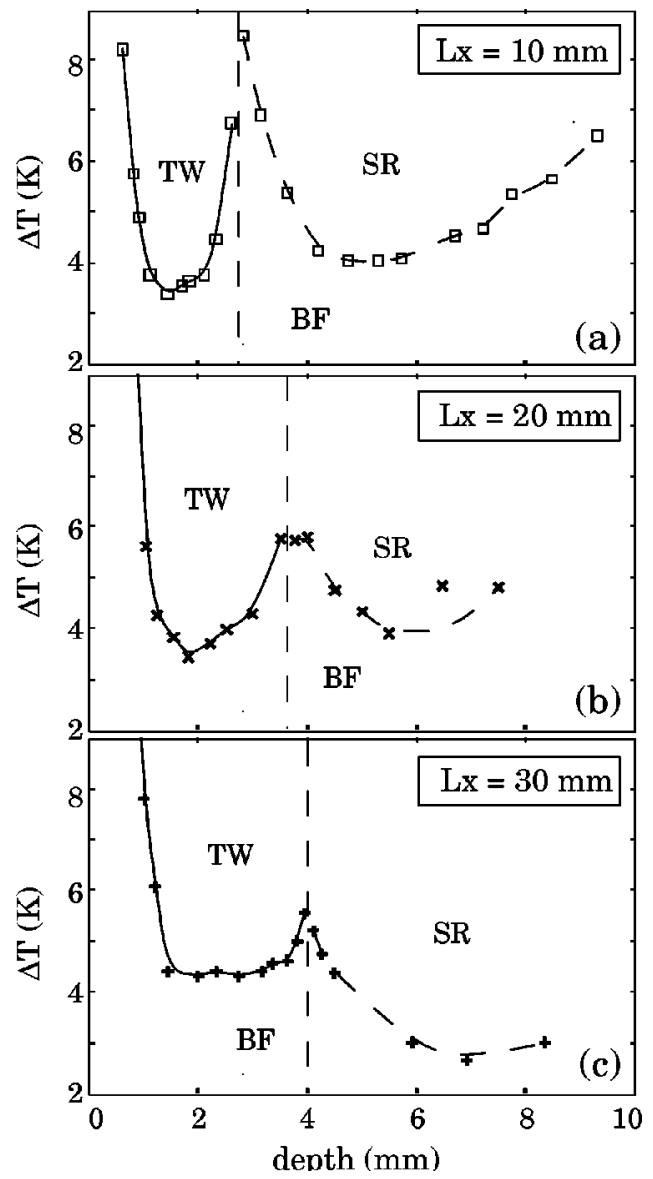

FIG. 2. Stability diagram: temperature difference $\Delta T_{c}$ vs height of liquid $h$ for (a) $L_{x}=10 \mathrm{~mm}$, (b) $L_{x}=20 \mathrm{~mm}$, and (c) $L_{x}=30 \mathrm{~mm}$. BF, TW, and SR, respectively, refer to basic flow, traveling waves, and stationary rolls. Vertical dashed lines correspond to the transitional depth $h_{r}$. 

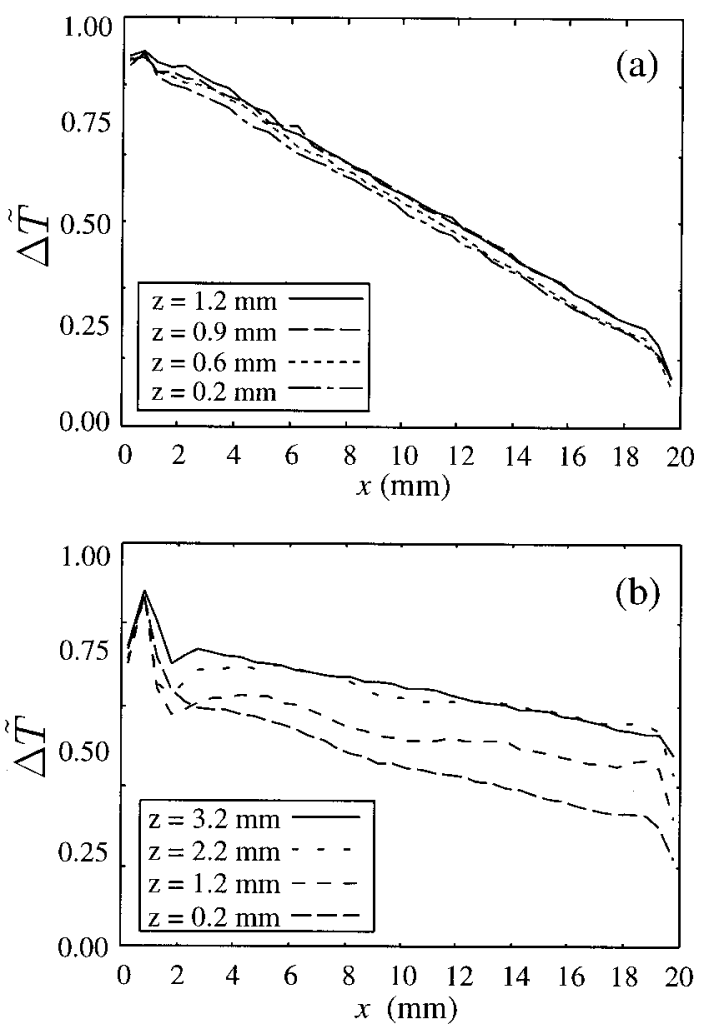

FIG. 3. Reduced temperature $\Delta \widetilde{T}=\left(T-T_{-}\right) / \Delta T$ vs $x$ for different depths $z$ for $L_{x}=20 \mathrm{~mm}$ and $\Delta T=3 \mathrm{~K}$, below the onset of traveling waves. (a) $h$ $=1.2 \mathrm{~mm}$, (b) $h=3.2 \mathrm{~mm}$.

The two parameters which control our experimental system are $h$, the height of fluid in the cell, and $\Delta T$, the horizontal temperature difference between the two walls. In fact, one must keep in mind that, when varying $h, \Gamma_{x}$ and $\Gamma_{y}$ are also varied. The experimental procedure was the following: For a given $L_{x}$ and height of fluid $h, \Delta T$ is increased from $\Delta T=0$ by steps and we wait for $t=30 \mathrm{~min} \sim 100 \mathrm{~h}^{2} / \nu$ at each step to allow the system to stabilize. Above the threshold, $\Delta T$ is then decreased to study the existence of hysteresis. The steps are smaller than $0.5 \mathrm{~K}$.

\section{RESULTS}

As soon as $\Delta T \neq 0$, a convective flow is created. The thermal gradient across the cell induces a surface tension gradient on the free surface of the fluid. Because of the thermocapillary effect, this gradient generates a surface flow from the hot side towards the cold side, with a bottom recirculation. Buoyancy forces also drives the flow in the same direction as thermocapillary forces. The basic flow appears thus as a long roll perpendicular to the gradient. In fact, depending on $\Gamma_{x}$ and $\Delta T$, a unicellular or a multicellular flow with transverse rolls can be observed (see below). This flow can then destabilize into different patterns, depending on the two parameters $\Delta T$ and $h$. The results are reported on the stability diagrams of Fig. 2 and show a dependence of the threshold values on $L_{x}$ and $h$. The region named basic flow (BF) accounts for both the steady unicellular and multicellular flow. Depending on the height of liquid, two regimes occur. When $\Delta T \geqslant \Delta T_{c}(h)$, for small $h$ values, the system

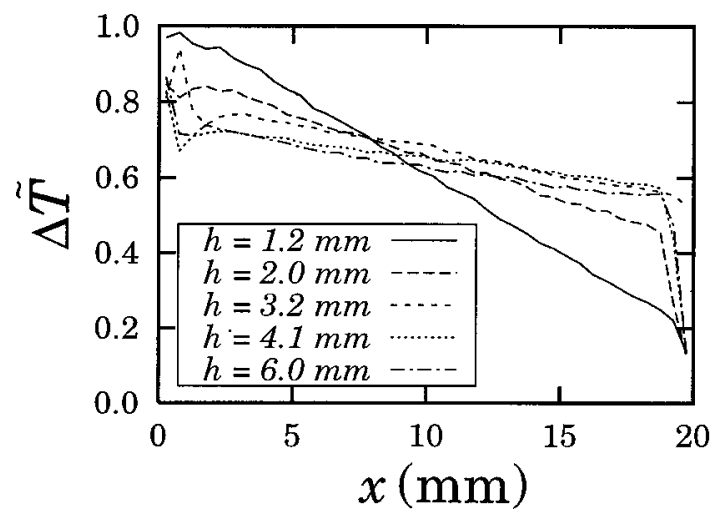

FIG. 4. Reduced temperature $\Delta \widetilde{T}$ vs $x$ on the free surface for different $h$, $L_{x}=20 \mathrm{~mm}$ and $\Delta T=3 \mathrm{~K}$, below the onset of traveling waves.

exhibits oblique traveling waves (TW), while for larger $h$ values, stationary longitudinal rolls (SR) are observed. The value $h=h_{r}$ which separates TW from SR increases with $L_{x}$ ( $h_{r}=2.7,3.6,4.0 \mathrm{~mm}$ for $L_{x}=10,20,30 \mathrm{~mm}$, respectively) while the threshold $\Delta T_{c}$ does not increase significantly. For each $h$, the reported $\Delta T_{c}$ value corresponds to the first observed pattern that invades the whole cell. In the following, we first present the basic flow and then the different patterns observed while varying $\Delta T$ and $h$.

\section{A. Basic flow}

The basic flow has been characterized through temperature measurements. Figure 3 presents the reduced horizontal temperature profiles $\Delta \tilde{T}(\mathbf{r}, t)=\left[T(\mathbf{r}, t)-T_{-}\right] / \Delta T$ obtained for $L_{x}=20 \mathrm{~mm}$ and for different depths in two different configurations: $h=1.2 \mathrm{~mm}$ and $h=3.2 \mathrm{~mm}$. For $h=1.2 \mathrm{~mm}$, the temperature profile is linear and gives a mean temperature gradient measured in the center of the container $\beta_{H}$ $=\partial T / \partial x$ which is of the order of the imposed temperature gradient $\beta_{i}=\Delta T / L_{x}$, i.e., $\beta_{H} \simeq 0.8 \beta_{i}$. Moreover, the profile is similar for all depths. This central temperature gradient $\beta_{H}$ defines for each depth $h$ an effective temperature difference calculated as $\Delta T_{H}=\beta_{H} \times L_{x}<\Delta T$. The difference between effective and imposed temperature difference is due to the

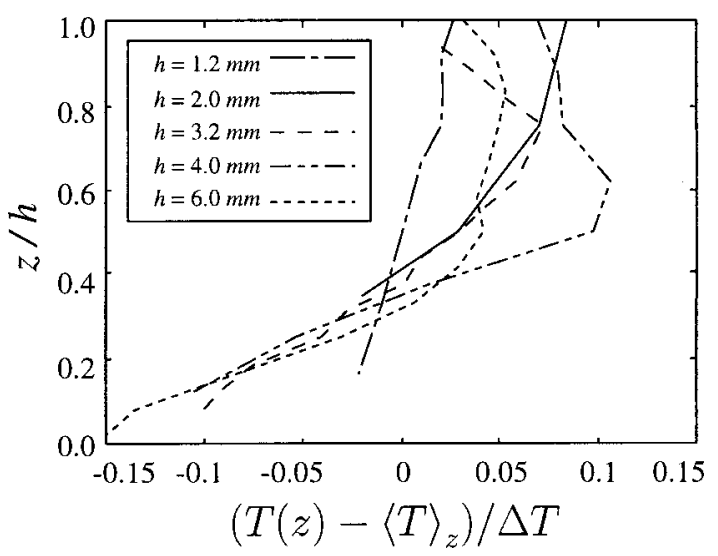

FIG. 5. Vertical temperature profiles for different $h$ for $L_{x}=20 \mathrm{~mm}$ and $\Delta T=3 \mathrm{~K}$ at $x=10 \mathrm{~mm}$ (middle of the container). $\langle T\rangle_{z}$ denotes the average of $T$ over $z$. 
presence of thermal boundary layers on both hot and cold sides. This effective gradient $\beta_{H}$ allows direct comparisons with theoretical analysis by Smith and Davis ${ }^{3}$ and Mercier and Normand ${ }^{9}$ who consider an infinite layer of fluid along $x$ (see below).

For $h=4 \mathrm{~mm}$, thermal boundary layers are much more important near the hot and cold walls and the measured temperature gradient is $\beta_{H} \simeq 0.2 \beta_{i}$. A local inversion of temperature can even be observed near the hot wall in the upper part of the fluid. This is an evidence of a roll in a narrow region near the hot wall. ${ }^{20,38}$ In fact, the ratio between the measured and the imposed temperature gradient $\beta_{H} / \beta_{i}$ continuously decreases when the height of liquid is increased (Fig. 4). When $h \geqslant 6 \mathrm{~mm}$, one can consider that there is no more temperature gradient in the middle of the cell; all the gradients take place in the vertical boundary layers.

The vertical temperature profiles obtained in the center of the cell for liquid layers with different height are presented in Fig. 5. For small height $(h=1.2 \mathrm{~mm})$, the vertical gradient is small and uniform in $z$. When $h$ increases, this gradient increases and becomes nonuniform along $z$ : A mean vertical temperature gradient $\beta_{V}$ can be defined. For $h$ above $3.2 \mathrm{~mm}$, an inversion of the vertical gradient is observed in the upper part of the fluid layer. Globally, when $h$ increases, $\beta_{H}$ decreases while $\beta_{V}$ increases. For the smallest $h$ (resp. highest) the gradient is nearly horizontal (resp. vertical). For high $h$, due to the return flow, we observe a vertical temperature gradient in the bottom of the cell. This profile is perhaps related to a downwards heat flux accross the glass bottom plate: Higher spatial resolution in the bottom boundary layer should be achieved to answer this question. The thermal boundary condition at the oil-air interface appears to be adiabatic for small $h$, while an upward-directed heat flux exists for greater $h$. This heat flux is at the origin of a sublayer below the surface which is unstable against BénardMarangoni type instability, the surface being colder than deeper in the fluid.

Depending on $\Delta T$ and on the aspect ratio $\Gamma_{x}$, a multicellular flow can be observed. As it was studied in details in
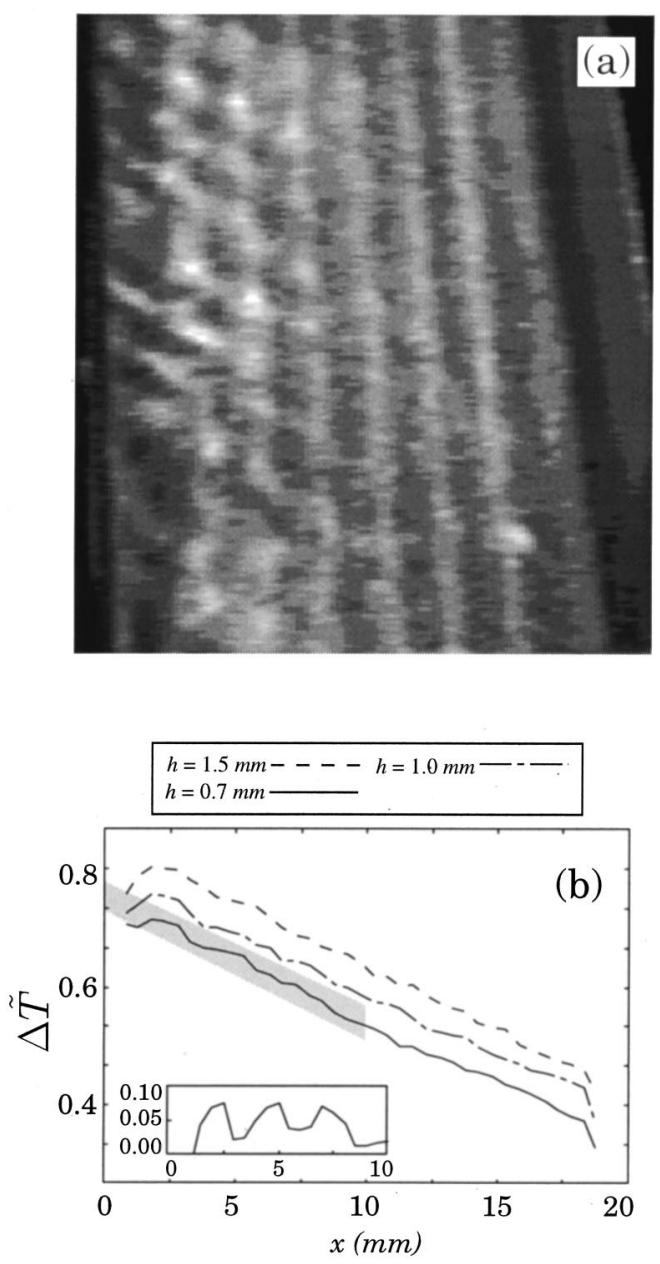

FIG. 6. Basic flow: Co-rotative rolls. (a) Upper view for $h=1 \mathrm{~mm}$ and $\Delta T=6 \mathrm{~K}$ visualized with iriodin particles; (b) horizontal temperature profiles for $h=2 \mathrm{~mm}$ and $\Delta T=3 \mathrm{~K}$. Inset: Temperature oscillations around the mean gradient in the gray marked region.

other works, ${ }^{14,19,28,37}$ we do not report a detailed study of this instability. Let us mention that visualizations revealed this flow to correspond to corotating rolls. They appear near the hot wall and their number decreases when $h$ increases $\left(\Gamma_{x}\right.$

TABLE III. Hydrothermal waves: Critical $\Delta T$ and critical Marangoni numbers vs fluid depth $h$ and dynamic Bond number $B o$ for $L_{x}=10,20$, and 30 mm. The Bond number has been computed with the classical definition: $B o(h)=(\rho g \alpha / \gamma) h^{2}$. The Marangoni number has been computed with $h$ and the global thermal gradient $\Delta T_{c} / L_{x}$ for the three values of $L_{x}$ and also with the measured horizontal gradient $\beta_{H}$ for $L_{x}=20 \mathrm{~mm}$. The gradient $\beta_{H}$ was measured for $\Delta T=3 \mathrm{~K}$ and $1.2 \leqslant h \leqslant 6 \mathrm{~mm}$ and the corresponding values have been interpolated.

\begin{tabular}{|c|c|c|c|c|c|c|c|c|c|c|c|c|c|}
\hline \multicolumn{4}{|c|}{$L_{x}=10 \mathrm{~mm}$} & \multicolumn{6}{|c|}{$L_{x}=20 \mathrm{~mm}$} & \multicolumn{4}{|c|}{$L_{x}=30 \mathrm{~mm}$} \\
\hline $\begin{array}{c}h \\
\mathrm{~mm}\end{array}$ & $\begin{array}{c}\Delta T_{c} \\
\mathrm{~K}\end{array}$ & $B o(h)$ & $M a\left(\Delta T_{c} / L_{x}, h\right)$ & $\begin{array}{c}h \\
\mathrm{~mm}\end{array}$ & $\begin{array}{c}\Delta T_{c} \\
\mathrm{~K}\end{array}$ & $\begin{array}{c}\beta_{H} \\
\mathrm{~K} \cdot \mathrm{mm}^{-1}\end{array}$ & $B o(h)$ & $M a\left(\Delta T_{c} / L_{x}, h\right)$ & $M a\left(\beta_{H}, h\right)$ & $\begin{array}{c}h \\
\mathrm{~mm}\end{array}$ & $\begin{array}{c}\Delta T_{c} \\
\mathrm{~K}\end{array}$ & $B o(h)$ & $\operatorname{Ma}\left(\Delta T_{c} / L_{x}, h\right)$ \\
\hline 0.60 & 8.21 & 0.04 & 760 & 0.84 & 10.01 & 0.500 & 0.09 & 908 & 908 & 1.00 & 7.80 & 0.12 & 668 \\
\hline 0.81 & 5.76 & 0.08 & 971 & 1.04 & 5.61 & 0.280 & 0.14 & 780 & 780 & 1.20 & 6.07 & 0.18 & 749 \\
\hline 0.90 & 4.90 & 0.10 & 1020 & 1.24 & 4.25 & 0.177 & 0.19 & 839 & 698 & 1.44 & 4.40 & 0.26 & 782 \\
\hline 1.10 & 3.77 & 0.15 & 1170 & 1.54 & 3.82 & 0.121 & 0.30 & 1170 & 736 & 1.98 & 4.30 & 0.49 & 1440 \\
\hline 1.43 & 3.38 & 0.26 & 1780 & 1.80 & 3.43 & 0.089 & 0.40 & 1430 & 739 & 2.32 & 4.40 & 0.67 & 2030 \\
\hline 1.70 & 3.55 & 0.36 & 2640 & 2.22 & 3.71 & 0.074 & 0.62 & 2350 & 932 & 2.74 & 4.30 & 0.94 & 2770 \\
\hline 1.82 & 3.63 & 0.41 & 3100 & 2.50 & 3.98 & 0.068 & 0.78 & 3200 & 1090 & 3.16 & 4.40 & 1.25 & 3770 \\
\hline 2.10 & 3.77 & 0.55 & 4280 & 3.00 & 4.29 & 0.058 & 1.12 & 4970 & 1340 & 3.36 & 4.54 & 1.41 & 4390 \\
\hline 2.32 & 4.46 & 0.67 & 6170 & 3.50 & 5.76 & 0.064 & 1.53 & 9070 & 2010 & 3.64 & 4.60 & 1.65 & 5220 \\
\hline 2.60 & 6.76 & 0.84 & 11700 & & & & & & & 3.80 & 5.00 & 1.80 & 6190 \\
\hline & & & & & & & & & & 3.93 & 5.54 & 1.93 & 7330 \\
\hline
\end{tabular}




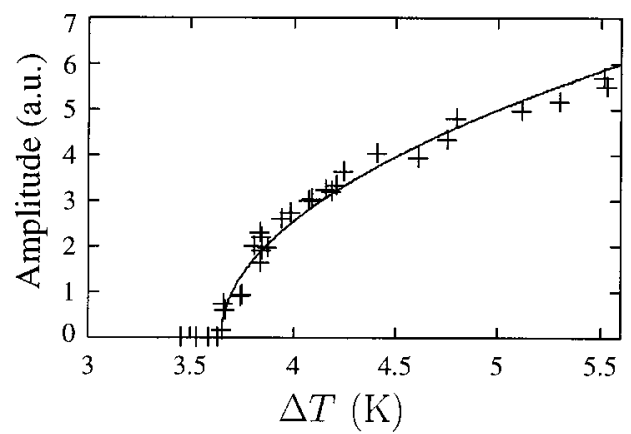

FIG. 7. Amplitude of the traveling waves vs $\Delta T$ for $h=1.7 \mathrm{~mm}, L_{x}$ $=10 \mathrm{~mm}$, and $L_{y}=180 \mathrm{~mm}$. Experimental data $(+)$ and fit $A=(\Delta T$ $\left.-\Delta T_{c}\right)^{1 / 2}$ (solid line).

decreases: 8 rolls for $h=1 \mathrm{~mm}$ and $L_{x}=20 \mathrm{~mm}$, two rolls for $h=6 \mathrm{~mm}$ ). These rolls can be visualized on an upper view of the cell (Fig. 6) and are observed as spatial oscillations on the horizontal temperature profiles. Different mechanisms have been proposed to account for this multicellular flow, ${ }^{9,13-15}$ but up to now, no complete answer has been given.

\section{B. Traveling waves}

Oblique traveling waves are observed for small depth layers $\left(h \leqslant h_{r}\right)$ for $\Delta T \geqslant \Delta T_{c} . \Delta T_{c}$ depends on $h$ (or $B o$ ): When increasing $h, \Delta T_{c}$ decreases from small $h$ towards a minimum and then increases up to $h_{r}$. The positions of the minimum and of $h_{r}$ increase with $L_{x}$ (Fig. 2 and Table III). As soon as the threshold is crossed, two waves which propagate in opposite direction along the $y$ axis, and towards the hot wall on the $x$ axis appear in the container, separated by a source. The source shape and its evolution with $\Delta T$ above the threshold depend of $h$.

The waves appear via a supercritical Hopf bifurcation: The frequency is finite at threshold ${ }^{1,23,26-28}$ and the amplitude of the waves behaves as $\left(\Delta T-\Delta T_{c}\right)^{1 / 2}$ (Fig. 7) $)^{32,39,40}$ (see Table IV). Using the amplitude critical behavior, a more accurate threshold value can be obtained, smaller than the one given by the observation of spatio-temporal diagrams. In fact, for $1.5 \mathrm{~mm} \leq h<h_{r}$ the transition for basic flow to traveling waves invading the cell is relatively sharp, occuring within a few tenth of a degree. However, for smaller depths of fluid, the oscillation that take place first are localized at the end boundaries. In order to study planar waves, we decided to report (Fig. 2, Table III) the onset in the long cells as the first state where the whole cell oscillates. This state can be as much as one or two degrees above the localized oscillation regime. This overestimation of the onset makes difficult the comparison with theory and experiments in shorter cells $^{27,28}$ (see Discussion).

\section{Wave sources}

Depending on the fluid depth $h$, two different types of sources of waves can be distinguished:

For smaller values of $h\left(h<h_{c}\right)$, the source looks like a point located on the cold wall and emits a circular wave. Due to the confinement by the channel in the $x$ direction, this wave becomes almost planar far from the source (Fig. 8).

(ii) For higher $h\left(h_{c}<h<h_{r}\right)$, the source looks like a line parallel to the $x$ axis and emits inclined planar waves. At onset, the pattern is symmetric and the source is in the middle of the channel. ${ }^{39}$ For $\Delta T$ slightly above threshold-typically within $0.3 \mathrm{~K}$-we observe the source to stand closer and closer to one end of the channel. Further from threshold, an asymptotic homogeneous state is reached, where just a single wave is present. This state can be seen as a source at one end which emits a wave that dies in a sink at the opposite end (Fig. 9). The present Paper focuses especially on these homogeneous patterns.

The transition between these two behaviors is not sharp. We observe a crossover domain inbetween and the transition is hysteretic when varying $h$. To illustrate this phenomenon, let us describe a constant $\Delta T=5.5 \mathrm{~K}$ experiment, i.e., slightly above onset, with $h$ slowly decreasing in the $L_{x}$ $=30 \mathrm{~mm}-L_{y}=180 \mathrm{~mm}$ cell. ${ }^{41}$ This experiment is illustrated in Fig. 10 by the position of the source (a), the period (b), and the wave number $k_{y}$ (c) measured far from the source and the transverse phase speed $c=\omega / k_{y}$ (d) which remains nearly constant when $h$ is varied. For $h>h_{c}=1.8 \mathrm{~mm}$ a single traveling wave is emitted by a source at the extremity of the channel. For $1.5<h<1.8 \mathrm{~mm}$ the source leaves the extremity and transforms progressively into a circular

TABLE IV. Nondimensional characteristics of the hydrothermal waves for some depths just above threshold: Projection of the wave vector along $y$ axis $k_{y}$, frequency $\omega=2 \pi / T$, and angle $\phi$.

\begin{tabular}{|c|c|c|c|c|c|c|c|c|c|c|}
\hline \multicolumn{3}{|c|}{$L_{x}=10 \mathrm{~mm}$} & \multicolumn{4}{|c|}{$L_{x}=20 \mathrm{~mm}$} & \multicolumn{4}{|c|}{$L_{x}=30 \mathrm{~mm}$} \\
\hline $\begin{array}{c}h \\
(\mathrm{~mm})\end{array}$ & $\begin{array}{c}k_{y} \\
(1 / h)\end{array}$ & $\begin{array}{c}\omega \\
\left(\nu / h^{2}\right)\end{array}$ & $\begin{array}{c}h \\
(\mathrm{~mm})\end{array}$ & $\begin{array}{c}k_{y} \\
(1 / h)\end{array}$ & $\begin{array}{c}\omega \\
\left(\nu / h^{2}\right)\end{array}$ & $\begin{array}{c}\phi \\
(\mathrm{rad})\end{array}$ & $\begin{array}{c}h \\
(\mathrm{~mm})\end{array}$ & $\begin{array}{c}k_{y} \\
(1 / h)\end{array}$ & $\begin{array}{c}\omega \\
\left(\nu / h^{2}\right)\end{array}$ & $\begin{array}{c}\phi \\
(\mathrm{rad})\end{array}$ \\
\hline 0.90 & 1.42 & 8.23 & 1.04 & 1.65 & 9.50 & 2.62 & 1.00 & 1.40 & 8.31 & 2.58 \\
\hline 1.10 & 1.19 & 7.72 & 1.24 & 1.22 & 7.82 & 2.62 & 1.20 & 1.52 & 9.23 & 2.65 \\
\hline 1.43 & 1.02 & 6.12 & 1.54 & 1.17 & 6.97 & 2.62 & 1.44 & 1.25 & 6.46 & 2.60 \\
\hline 1.82 & 1.47 & 9.05 & 1.80 & 1.10 & 7.18 & 2.29 & 1.98 & 0.80 & 6.15 & 2.40 \\
\hline \multirow[t]{4}{*}{2.32} & 1.63 & 11.02 & 2.22 & 1.41 & 9.30 & 2.23 & 2.32 & 0.72 & 6.15 & 2.09 \\
\hline & & & 2.50 & 1.32 & 9.56 & 2.16 & 2.74 & 1.05 & 10.62 & 1.96 \\
\hline & & & 3.00 & 1.43 & 15.51 & $\sim 1.8$ & 3.16 & 1.15 & 12.62 & $\sim 1.75$ \\
\hline & & & & & & & 3.93 & 1.01 & 15.23 & $\sim 1.75$ \\
\hline
\end{tabular}



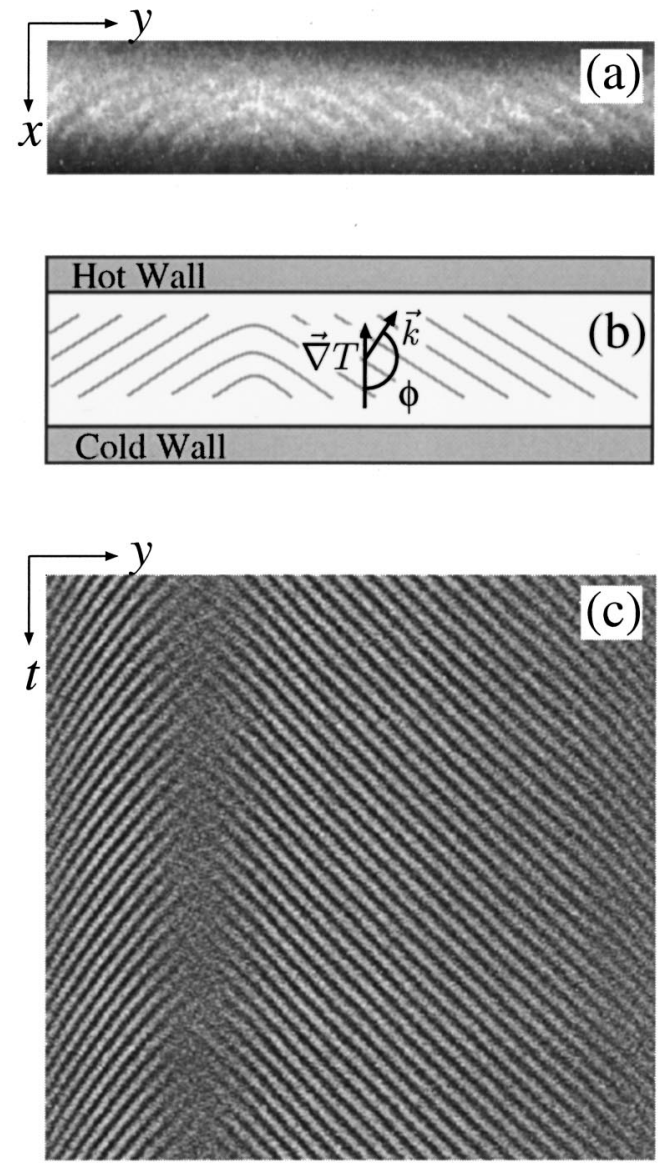

FIG. 8. Traveling waves, $h<h_{c}$. (a) Shadowgraphic image and (b) schematic drawing of the pattern in an horizontal plane; (c) spatiotemporal evolution for $L_{x}=30 \mathrm{~mm}, L_{y}=250 \mathrm{~mm}, h=1.6 \mathrm{~mm}$, and $\Delta T=5.6 \mathrm{~K}$. The field of view corresponds to $\sim 185 \mathrm{~mm}$.

source. At $h=1.5 \mathrm{~mm}$, we observe a discontinuity of the wave number and frequency. Below this value the source is perfectly circular and continue to move towards the center of the channel. Finally the waves disappear at $h=0.90 \mathrm{~mm}$. The crossover between the two behaviors can be tracked on spatiotemporal data: For a source of circular waves, the projection of the local wave vector along the $y$ direction changes continuously from a negative value to a positive one when crossing the source; whereas for source of planar waves, we observe a superposition of waves with opposite wave number $k_{y}$. The superposition occurring in the latter case results in the existence of standing waves (along $y$ axis) in the core of the source. So we observed the amount of standing waves to smoothly decrease by a factor 10 when $h$ decreases from 1.8 to $1.5 \mathrm{~mm}^{41}$ When performing the reverse experiment and increasing $h$, the source remains located away from the extremities in a small hysteretic region above $h_{c}$. No systematic study of this transition with parameters $h$ and $\Delta T$ has been performed. We have observed that $h_{c}$ increases with $L_{x}: h_{c}=1.1,1.6,1.8 \mathrm{~mm}$ for $L_{x}=10,20,30 \mathrm{~mm}$, respectively.

Other sources and sinks may even appear in the cell when strong increases of $\Delta T$ are made, but they annihilate, leaving a single source. Far above $\Delta T_{c}$ they may even remain stable, like frozen. The asymptotic behaviors described
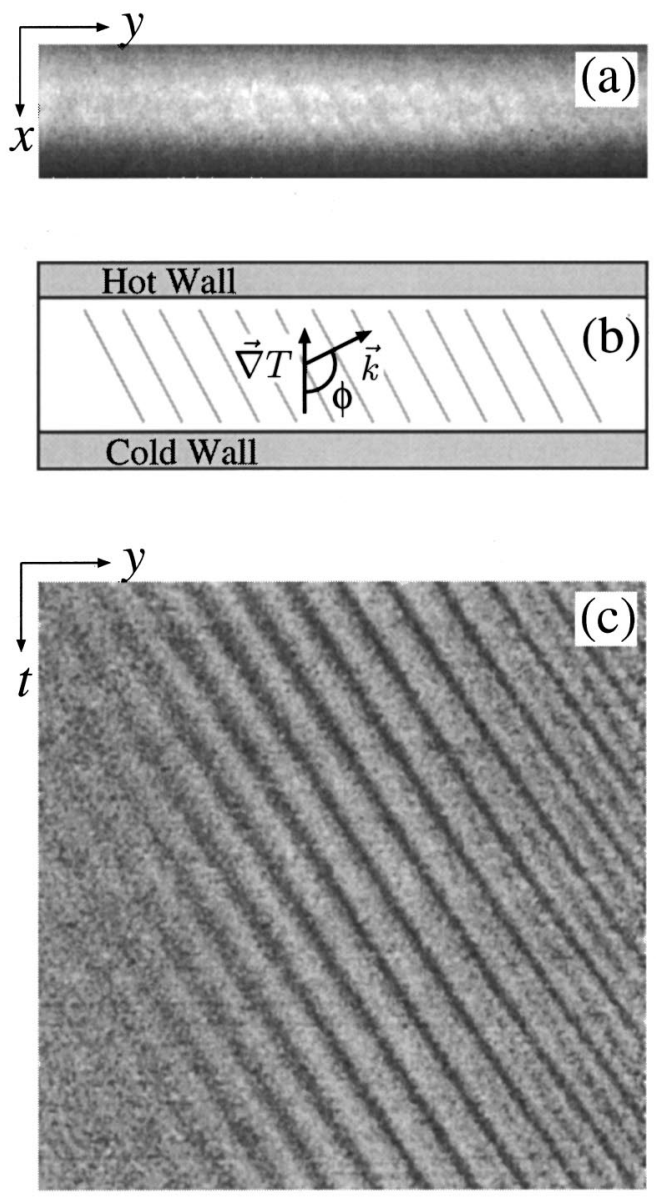

FIG. 9. Traveling waves, $h_{c}<h<h_{r}$. (a) Shadowgraphic image and (b) schematic drawing of the pattern in an horizontal plane; (c) spatiotemporal evolution for $L_{x}=30 \mathrm{~mm}, L_{y}=250, h=3.00 \mathrm{~mm}$, and $\Delta T=4.64 \mathrm{~K}$. The field of view corresponds to $\sim 185 \mathrm{~mm}$.

above concern thus a narrow band above $\Delta T_{c}$. Finally, in the periodic annular channel- $L_{x}=10 \mathrm{~mm}, L_{y}=503 \mathrm{~mm}$ - the two types of sources are also observed above and below $h_{c}$, but only during transients; sources and sinks are both unstable near onset always leaving a single wave. We can thus conclude about the stability of defects that sources are stable at small heights in the rectangular container and sinks are always unstable except at the extremities of the channel.

The two types of sources reported above can be related to the two types of hydrothermal waves observed in a larger aspect ratio experiment. ${ }^{37,40}$ However, in our very long rectangular channel, the two type of wave can only be differentiated close to the source.

\section{Temperature oscillations measurements}

Temperature measurements reveal that the traveling waves correspond to bulk temperature oscillations. As shown in Fig. 11, the corresponding temperature evolution for a defined position $x_{0}$ can be described as $T_{x_{0}}(z, t)$ $=\widetilde{A}(z) \cos \left(\omega_{T} t\right)+\left\langle T\left(x_{0}, z\right)\right\rangle$, where $\omega_{T}$ is the frequency of the oscillation, identical to that measured with shadowgraphy $\omega, \widetilde{A}(z)$ is the amplitude of the oscillation for each depth and $\langle T(x, z)\rangle$ denotes a time-average of the temperature signal in 


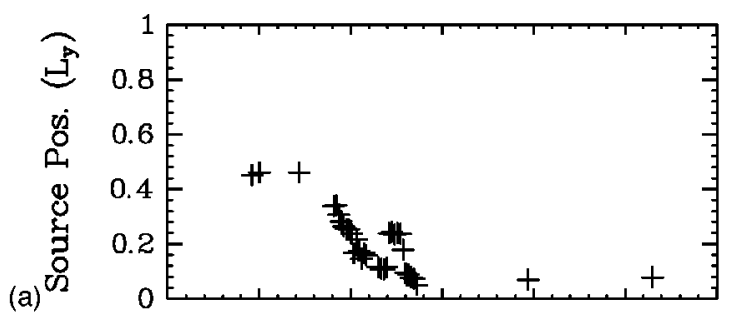

(b)

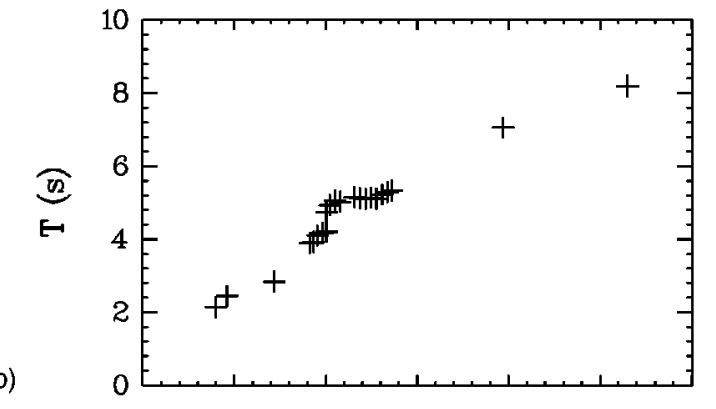

(c)

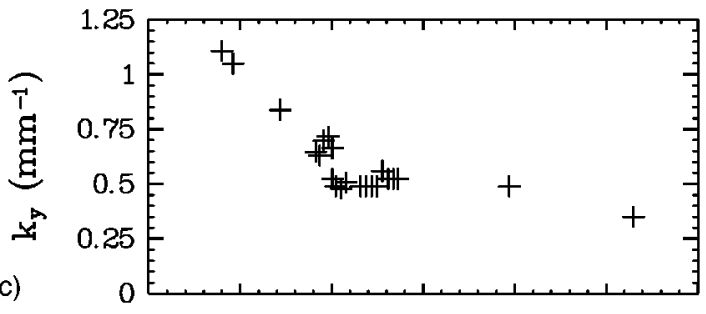

(d)

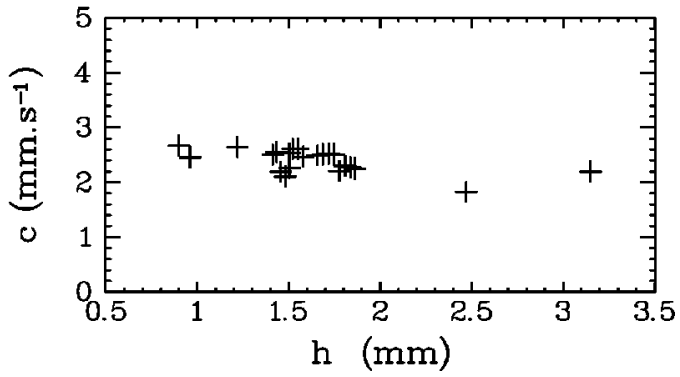

FIG. 10. Evolution with varying $h$ at $\Delta T=5.5 \mathrm{~K}$ for $L_{x}=30 \mathrm{~mm}$ and $L_{y}$ $=180 \mathrm{~mm}$. (a) $y$ position of the source in the cell. (b) Period $T$ of the wave. (c) Projection of the wave vector $k_{y}$ away from the source. (d) Projection of the phase velocity $c=\omega / k_{y}$ along $y$.

a given spatial position $(x, z)$. This amplitude is less than 0.5 $\mathrm{K}$, and its $z$-dependence can be seen in Fig. 11(b).

The maximum of the amplitude of the oscillations is reached at middle-depth $(z \simeq h / 2)$, and the minimum at the surface $(z=h)$. This kind of behavior was predicted by Smith $^{5}$ and experimentally observed in another configuration by Pelacho et al. ${ }^{28}$ This temperature behavior is compatible with the proposed hydrothermal wave instability mechanisms, which is responsible of the up-flow component of the wave number.

\section{Characteristics of the waves at threshold}

The waves behave as plane waves near the threshold, i.e., an unique wave number and frequency can describe their evolution. As the wave propagates from the cold to the hot side, the $x$ component $k_{x}$ is negative in our referential, and $k_{y}$ is positive (resp. negative) for the right (resp. left) traveling
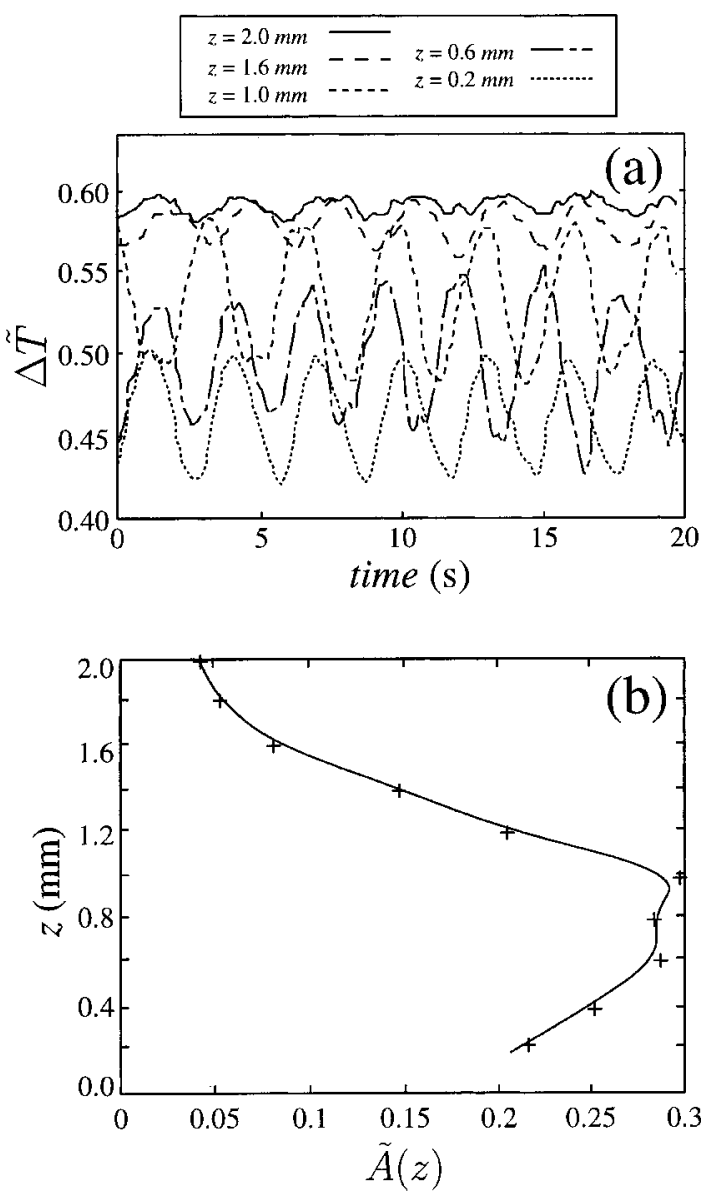

FIG. 11. Traveling waves for $h=2 \mathrm{~mm}$ and $\Delta T=6 \mathrm{~K}$. (a) Evolution of the reduced temperature $\Delta \widetilde{T}$ with time at different depths $z$. (b) Amplitude $\widetilde{A}(z)$ of the waves vs depth $z$.

wave. As a consequence, $\boldsymbol{k}$ forms an angle $\phi$ (see schematics in Figs. 8 and 9) with the $x$ axis, varying between $\pi / 2<\phi$ $<\pi$.

When we change the experimental parameters, the wave number $k$, the angle $\phi$ and the frequency $\omega$ evolve. For the three values $L_{x}=10,20,30 \mathrm{~mm}$ two regions can be distinguished:

(i) For $h \leqq h_{c}\left(L_{x}\right)$, the wave number $k_{y}$ and the frequency $\omega$ decrease with $h$ while the angle has a nearly constant value $\phi \sim 0.83 \pi=2.6 \mathrm{rad}$ (Fig. 12). Note that if we define the angle with the gradient as $\alpha=\pi$ $-\phi$, his value is approximatively $\alpha \sim \pi / 6$.

(ii) For $h_{c} \leqq h<h_{r}$ the frequency increases with $h$, but the angle decreases until a value $\phi=0.64 \pi \sim 2 \operatorname{rad}(\alpha$ $\sim \pi / 3$ for large $h$, Fig. 12). The wave number $k_{y}$ increases slightly for $h \geq h_{c}$ and saturates when approaching $h_{r}$.

The different characteristics of the waves for some values of $h$ are summarized in Table IV.

\section{Influence of the aspect ratio}

To evaluate the influence of the aspect ratio $\Gamma_{y}=L_{y} / h$ or $\Gamma=L_{y} / L_{x}$ on the instability, various experiments were real- 

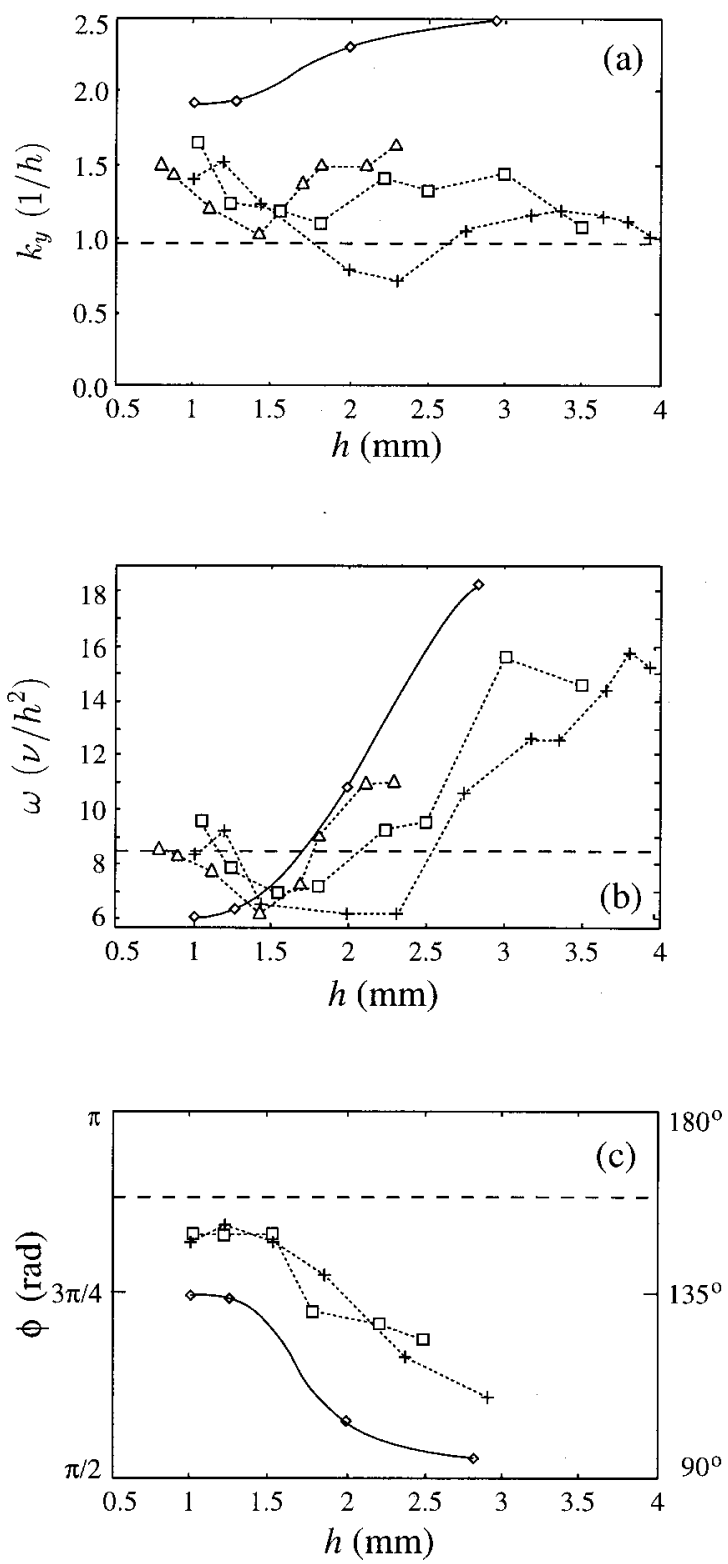

FIG. 12. Traveling waves just above threshold: Evolution of (a) the wave number $k_{y}$, (b) the frequency $\omega=2 \pi / T$, (c) the angle $\phi$, as a function of $h$. Experimental data (dotted lines): $(\triangle) L_{x}=10 \mathrm{~mm},(\square) L_{x}=20 \mathrm{~mm},(+)$ $L_{x}=30 \mathrm{~mm}$. Theoretical predictions: (dashed line) Smith and Davis (Ref. 3); ( $\diamond$ and solid line) Mercier and Normand (Ref. 9).

ized for $0.75<h<2.75 \mathrm{~mm}$ and various $L_{y}$ between 30 and $90 \mathrm{~mm}$, leaving $L_{x}=30 \mathrm{~mm}$ constant, i.e., for horizontal aspect ratio $\Gamma$ from 1 to 3 .

For small height, between 0.75 and $1 \mathrm{~mm}$, we observe for $\Gamma \sim 1,2$, and 3 the waves to nearly propagate along the direction of the thermal gradient from cold side to hot side. This corresponds mainly to the core of point sources as described in Sec. III B 1. A perfectly symmetric point source for $L_{y}=90 \mathrm{~mm}$ is shown in Fig. 13 .

For higher height, between 1.75 and $2.75 \mathrm{~mm}$, we observe for $\Gamma \sim 1\left(L_{y}=30 \mathrm{~mm}\right)$ and $\Gamma \sim 3\left(L_{y}=90 \mathrm{~mm}\right)$, planar waves to propagate obliquely from cold side to hot side along $x$ axis and both side along $y$ axis. Such behavior recalls precisely the observations in longer cells. However, for

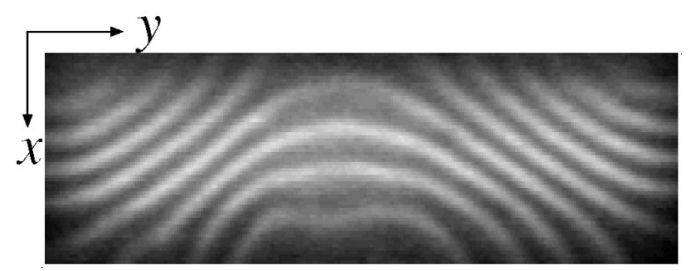

FIG. 13. Shorter cell: Shadowgraphy snapshot for $h=1.0 \mathrm{~mm}, L_{x}$ $=30 \mathrm{~mm}, L_{y}=90 \mathrm{~mm}(\Gamma=3)$ and $\Delta T=7.3 \mathrm{~K}$. The cold side is down. This nearly circular wave propagates from the point source located in the middle of the cold side. Menisci are very important in distorting the sides of the pattern outside the cell. The whole cell is visible.

$L_{y}=50 \mathrm{~mm}, h=2.75 \mathrm{~mm}$, and $\Delta T=5.8 \mathrm{~K}>\Delta T_{c}$, we report an inverse dynamics. Traveling waves are observed which propagate from the hot to the cold side, along the gradient direction, and are reminiscent of those observed by Ezersky et $a ._{.}{ }^{21,22}$ and Garcimartín et al. ${ }^{20}$

Thresholds have been measured for $L_{y}=90 \mathrm{~mm}$ to be just a few tenth of a degree above those presented above for long channels. A discussion concerning the different thresholds measurement methods is presented in Sec. IV C. For shorter cells, no accurate measurements have been carried out but the threshold appears generally above those for longer channel configuration. This finite size effect as been recently studied by Pelacho et al. ${ }^{42}$

Let us note that for small aspect ratio cells the relative importance of menisci with respect to the bulk grows. For low $h$ especially, because $\Delta T_{c}$ is very high, oscillatory instabilities appear in the menisci for $\Delta T$ below the hydrothermal-waves onset in the bulk.

\section{Stationary rolls}

For $h>h_{r}$ the basic flow destabilizes in the form of a stationary pattern with a wave vector perpendicular to the horizontal applied gradient. At threshold, the pattern appears only near the hot side and invades the rest of the cell when $\Delta T$ is increased. A typical shadowgraphy of the cell is shown in Fig. 14. These stationary patterns correspond to the destabilization of the primary roll in the $y$ direction as in BénardMarangoni convection. The wave number associated with this pattern remains nearly constant $(k \simeq 3.2)$ for a large range of values of $h \geqslant h_{r}$ and increases slowly for larger $h$ (Fig. 15).

Using iriodin particles, we have visualized the flow inside these convective cells: It consists in a superposition of a circulation in the $x z$ plan due to the basic flow and a convective circulation in the $y z$ plan for each cell due to bulk induced convection. The walls of each cell are well defined,

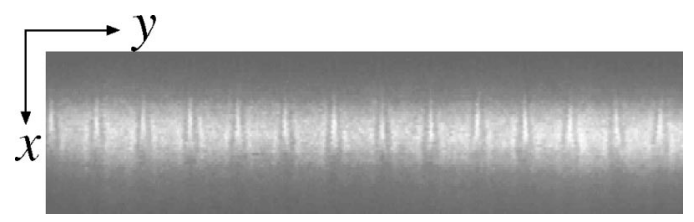

FIG. 14. Stationary rolls, $h=6 \mathrm{~mm}, \Delta T=6 \mathrm{~K}, L_{x}=30 \mathrm{~mm}$. Shadowgraphic image of the pattern in a horizontal plane. The cold side is down. 


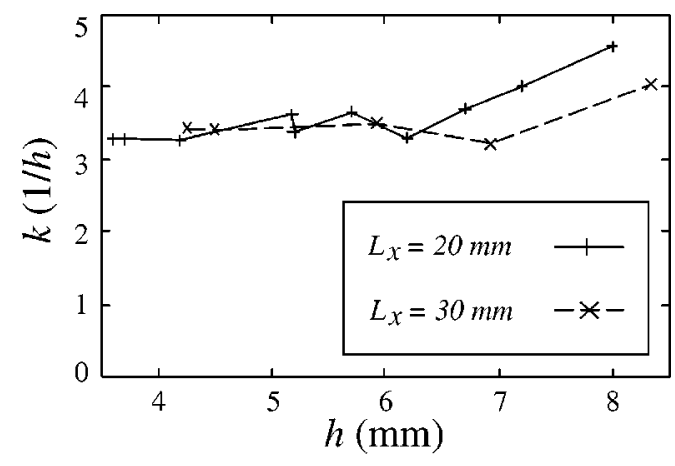

FIG. 15. Stationary rolls: Evolution of the wave number $k$ as a function of the depth of liquid $h$ at threshold $\left(\Delta T=\Delta T_{c}\right)$.

and the fluid does not cross them. In a convective cell, because of the primary roll flow, the fluid moves upwards near the hot wall, and downwards near the cold wall.

For intermediate $x$, the observation of independent cells along the $y$ axis allows to describe the temperature profiles in Fig. 16 as two superimposed layers consisting of counterrotating rolls. Because of the convective flow, the fluid circulates in the $y z$ plan, ascending in some places, and descending in others. These recirculations induce temperature modulations along the $z$ direction inside the upper $(2<z$ $<6 \mathrm{~mm})$ and lower $(0<z<2 \mathrm{~mm})$ layers (Fig. 16). An isotherm $(z \sim 2 \mathrm{~mm})$ separates both layers.

The vertical temperature profile below onset (Fig. 5) exhibits two layers: An upper layer near the surface which is unstable with respect to Bénard-Marangoni criterion and a lower layer with stable thermal stratification. Linear stability analysis of uniformly heated layer of depth $d$ predicts a nondimensional wave number $k \sim 2$ in Bénard-Marangoni convection. ${ }^{43}$ In the $d=2 h / 3$ upper layer, this would lead to $k \simeq 3$, close to the $k=3.2$ measured value.

\section{Further from threshold}

Further from threshold, the different patterns destabilize:

At small depth $h \lesssim h_{c}$, we observe series of traveling holes $^{33}$ to be shed continuously in the intermediate

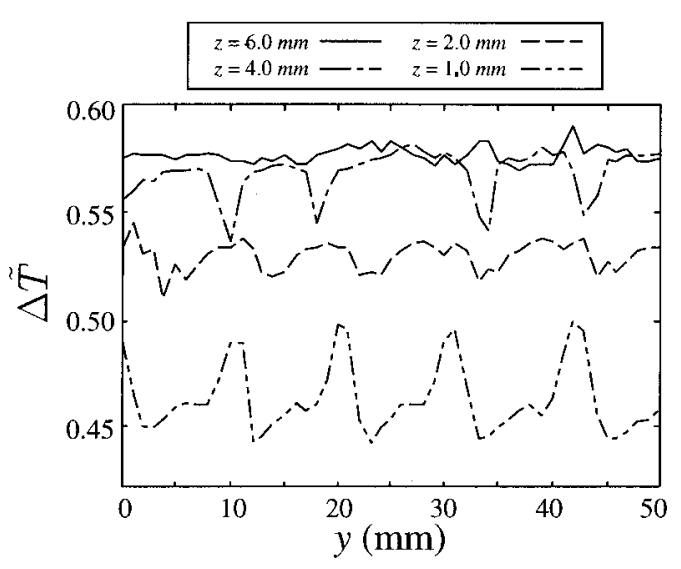

FIG. 16. Stationary rolls: Horizontal reduced temperature profiles $\Delta \widetilde{T}$ vs $y$ for different depths $z$ for $x=10 \mathrm{~mm}, h=6 \mathrm{~mm}$, and $\Delta T=10 \mathrm{~K}$. Those profiles have not been recorded simultaneously and might be shifted.

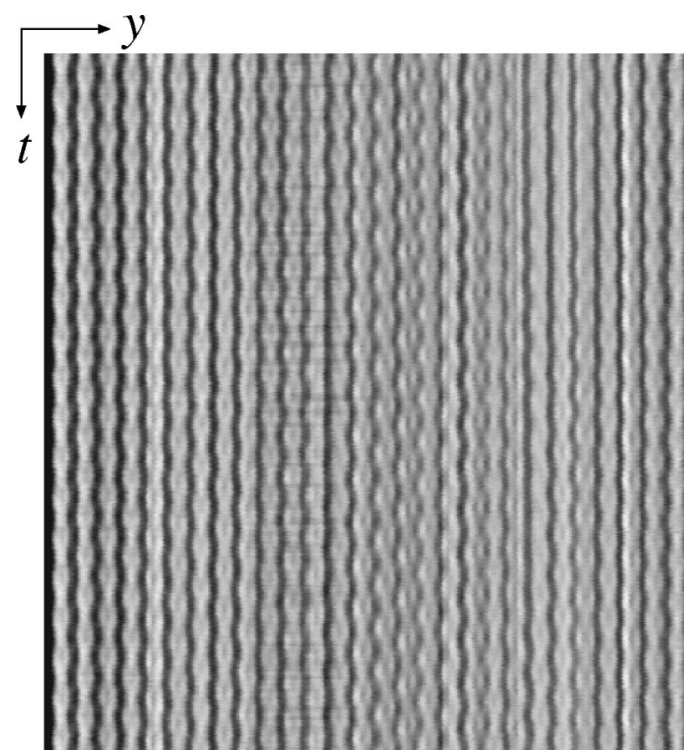

FIG. 17. Spatiotemporal evolution for stationary rolls: Optical mode for $L_{x}=20 \mathrm{~mm}, h=4 \mathrm{~mm}$, and $\Delta T=13 \mathrm{~K}$ far from threshold.

regions located between the circular source and the planar-wave regions. Whereas there is almost no defect near onset, their number increases with $\Delta T$ (cf. Fig. 2 of Burguete et al. ${ }^{33}$ ).

(ii) For intermediate depth, $h_{c} \leqslant h<h_{r}$, we report the occurrence of a secondary instability in the shape of modulational instability of the single-wave-pattern. This instability is similar to Eckhaus instability but occurs with low but finite wave number. We observe spatially growing wave number-modulations which eventually lead to regular shedding of spatio-temporal dislocations in the cell. This patterns are quasiperiodic in both frequency and space. Downstream the dislocations, a lower-wave number pattern is observed, which is either monoperiodic or chaotic owing to $h$ and $L_{x}$. These results will be reported elsewhere.

(iii) For large depth $h>h_{r}$, the stationary pattern has a secondary instability in the form of an oscillation in phase-opposition (optical mode, Fig. 17) with a finite frequency at threshold. This behavior has been observed in similar steady pattern for other convection experiments: Rayleigh-Benard convection in narrow gap, ${ }^{44}$ and in nonuniform heating, ${ }^{45}$ where a fluid layer is heated from the bottom with a nonuniform temperature profile.

\section{E. Higher Prandtl number fluid}

Finally, a restricted set of experiences using a $P=30$ ( $\nu=2 \mathrm{cSt}$ ) fluid has been realized to evaluate the influence of the Prandtl number. At $h=2.0 \mathrm{~mm}$ no hydrothermal waves were observed up to $\Delta T=30 \mathrm{~K}$. For $h>3 \mathrm{~mm}$, stationary rolls where observed. According to available experimental results in shallow horizontal (rectangular or annular) layers hydrothermal waves have not been reported above $P=17$. This annular experiment in ethanol ${ }^{23}$ has shown hydrothermal waves as well as surface waves depending of $h$. Never- 
theless Priede and Gerbeth ${ }^{10}$ predicted waves up to $P$ $=1000$. We believe this prediction to be correct, but it is experimentally very difficult to produce the critical gradient. The higher the Prandtl number, the sharper the temperature variation in the thermal boundary layers at both extremities: The effective horizontal gradient $\beta_{H}$ keeps small even for high $\Delta T$.

\section{DISCUSSION}

\section{A. Relevant parameters}

\section{Spatial scales and Bond number}

The experimental data show the existence of two transition depths $h_{c}$ and $h_{r}$. These depths separate three regions where different forces dominate. For small $h$, surface stresses are dominant and no vertical stratification has been observed (Fig. 5). Buoyant effects are small. Above $h_{c}$, buoyant forces are no more negligible, and the temperature profile varies with $z$ (Fig. 5). Finally, above $h_{r}$ the buoyant forces dominate and the thermal gradient is almost vertical.

The stability diagrams-minima positions, $h_{c}, h_{r}$ (Fig. 2) - and the characteristics of the waves and of the stationary rolls- $k, f, \phi$ (Figs. 12 and 15)-move towards larger depths when $L_{x}$ increases. We tried to reduce the data for different $h$ and $L_{x}$. The natural idea is to plot the data vs the Bond number $B o=R a / M a$. With the classical reduction ${ }^{9}$ $\beta=\Delta T / L_{x}$ and $d=h$ - the Bond number varies as $h^{2}$ and does not reduce the data. Other combinations of $h$ and $L_{x}$ have been tested and $h^{3} / L_{x}$ gives the best results: All these curves collapse into single ones (Figs. 18 and 19). In particular the transition between hydrothermal waves and stationary rolls occurs for $h_{r}^{3} / L_{x}=2.1 \pm 0.2 \mathrm{~mm}^{2}$.

As far as the Bond number is believed to be the relevant parameter for this flow, our experimental results impose $B o$ to be a function of $h^{3} / L_{x}$. A very simple way to obtain this is to build the Marangoni number using exclusively the horizontal scale $L_{x}$ and the Rayleigh number using exclusively the depth $h$

$$
M a\left(\frac{\Delta T}{L_{x}}, L_{x}\right)=\frac{\gamma \Delta T L_{x}}{\rho \kappa \nu}, \quad R a\left(\frac{\Delta T}{h}, h\right)=\frac{g \alpha \Delta T h^{3}}{\kappa \nu} .
$$

The Bond number is then

$$
B o\left(h, L_{x}\right)=\frac{\rho g \alpha}{\gamma} \frac{h^{3}}{L_{x}} .
$$

For the transitional depth $h_{r}$, we compute $B o\left(h_{r}, L_{x}\right)$ $=0.25$ showing buoyant and capillary forces to be of the same order of magnitude.

\section{Thermal gradient}

Let us note that even if the experiment control parameter is the horizontal temperature difference $\Delta T$ this study has shown the necessity to measure both horizontal and vertical effective gradients $\beta_{H}$ and $\beta_{V}$ to understand the instability mechanisms.

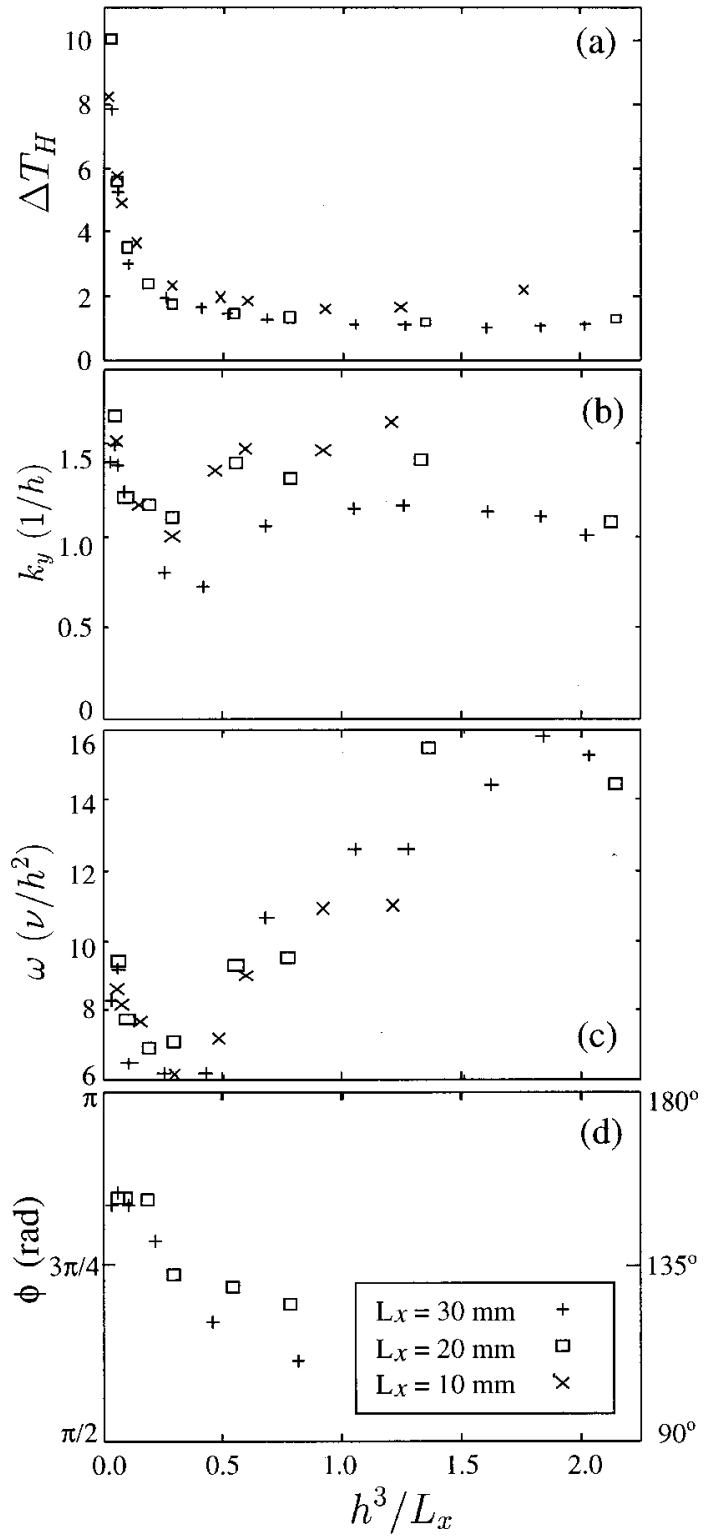

FIG. 18. (a) Stability diagram in reduced coordinates: Effective horizontal temperature difference $\Delta T_{H}=\beta_{H} L_{x}$ vs $h^{3} / L_{x}$ for different $L_{x}$. Evolution of (b) the wave number $k_{y}$, (c) the frequency $\omega=2 \pi / T$, and (d) the angle $\phi$ as a function of $h^{3} / L_{x}$.

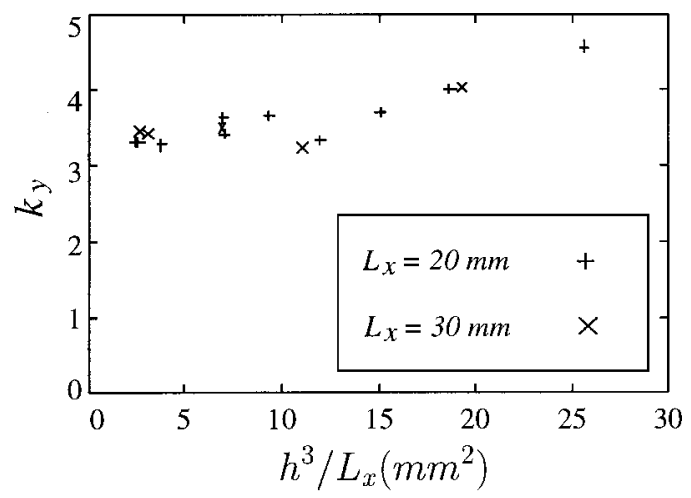

FIG. 19. Stationary rolls: Evolution of the wave number $k$ at threshold as a function of $h^{3} / L_{x}$. 


\section{Biot number}

In infinite layer theoretical studies, the Biot number is used to control the vertical temperature profile. In our experiment, the temperature profiles have been measured and shown to vary along $x$. Also, the air layer is typically $1 \mathrm{~cm}$ thick and is, therefore, animated by strong convective flows. These two effects make it impossible to calculate an exact value of $B i$. It could be possible to measure it from very careful measurement of the temperature field in the air very near the fluid surface.

Using our data, the only possibility is to compare the heat flux across the free surface from vertical temperature profiles (Fig. 5): The effective Biot number increases with $h$.

\section{B. Comparison with theoretical results}

\section{Hydrothermal waves}

In this section, we will compare our measurements with different theoretical results. We will use the classical definitions of Marangoni and Bond numbers, $\mathrm{Ma}^{T h}$ and $\mathrm{Bo}^{T h}$

$$
M a^{T h}=M a\left(\frac{\Delta T}{L_{x}}, h\right)=\frac{\gamma}{\rho \kappa \nu} \frac{\Delta T h^{2}}{L_{x}}, \quad B o^{T h}=\frac{\rho g \alpha}{\gamma} h^{2} .
$$

Furthermore, as far as theoretical studies concern only planar waves, we compare them with experimental data collected far from the sources where waves are planar whatever $h$.

Because the thermocapillar effects dominate for small $h$, we expect the results obtained by Smith and Davis ${ }^{3,4,11}$ to describe our experimental results. The nondimensional characteristics predicted by Smith and Davis for the return flow using a fluid with $P=10$ and $B i=0$ can be summarized as: Wave number $|k| \sim 2.5$, propagation angle $\phi \sim 160^{\circ}$, frequency $\omega \sim 0.14$ and phase velocity $c \sim 0.06$. It is important to note that the time unit used by Smith and Davis is $\nu \rho / \gamma \beta_{H}$. These waves appear for a critical Marangoni number $M a_{c}^{T h}=300$.

Let us consider the case $L_{x}=30 \mathrm{~mm}$. (The data presented in Table IV for $L_{x}=10$ or $20 \mathrm{~mm}$ give similar results.) For $h<h_{c}$, a very good agreement with SD is found. For example, for $h=1.00 \mathrm{~mm}$, and using the Smith and Davis time unit definition, the experimental values are $|k|=2.6$, $\phi=150^{\circ}, \omega=0.14$, and $c=0.05$. Nevertheless, the experimental critical Marangoni number value is $M a_{c}^{T h}=668$. This value is larger than the predicted value but corresponds to the invasion of the whole cell by the waves. We studied the first occurence of waves in shorter cells allowing a complete visualization and plot the observed threshold in the $(\mathrm{Ma}, \mathrm{Bo})$ plane in Fig. 20. We report $M a^{T h}=434$ for the same depth with $L_{y}=180 \mathrm{~mm}$. The variation of the critical Marangoni number with $B o$ is in very good agreement with the predicted limit $M a_{c}^{T h}=300$ for $B o=0$.

On the other side, for $h=2.74 \mathrm{~mm}>h_{c}$, the wave appear for $M a_{c}^{T h}=2800$ which reduces to 840 when based on the estimated effective horizontal temperature gradient $\beta_{H}$. The experimental characteristics of the waves above onset are: $|k|=1.1, \phi=110^{\circ}, \omega=0.18$, and $c=0.16$. A discrepancy is found for $|k|, \phi$ and $\omega$ between the experimental values and these values predicted by SD.

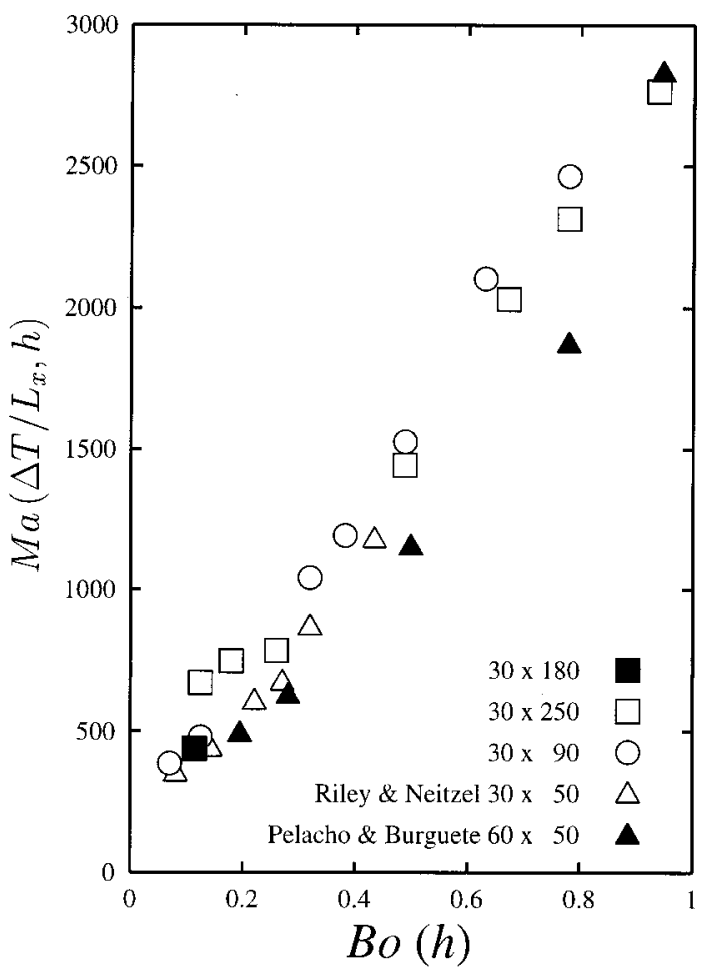

FIG. 20. Critical Marangoni $\operatorname{Ma}\left(\Delta T_{c} / L_{x}, h\right)$ number vs dynamic Bond number $B o(h)$. Open squares stand for $L_{x}=30 \mathrm{~mm}$ and $L_{y}=250 \mathrm{~mm}$ data reported in Table III. The closed squares stands for a measurement in the $L_{x}=30 \mathrm{~mm}$ and $L_{y}=180 \mathrm{~mm}$ cell and the open circles for the $L_{x}=30 \mathrm{~mm}$ and $L_{y}=90 \mathrm{~mm}$ cell. Open triangles stand for Riley and Neitzel's data (Ref. $27)$ in $L_{x}=30 \mathrm{~mm}$ and $L_{y}=50 \mathrm{~mm}$ cell and closed triangles stand for Pelacho and Burguete's data (Ref. 28) in $L_{x}=60 \mathrm{~m}$ and $L_{y}=50 \mathrm{~mm}$ cell. See text for details.

Mercier and Normand ${ }^{9}(\mathrm{MN})$ have considered the effect of bulk forces. For $h \sim 1 \mathrm{~mm}$ (small $B o$ ) their results are similar to SD. For $B o^{T h}=1 \quad(h=2.83 \mathrm{~mm}), P=7$ and $B i$ $=1$ they report $M a_{c}=386$. Using as time unit $h^{2} / \nu$, they found: $k \sim 2.5, \phi \sim 100^{\circ}, \omega \sim 19, c \sim 7$. Using this time unit, the experimental values for $h=2.74 \mathrm{~mm}$ become: $k=1.1$, $\phi=110^{\circ}, \omega=11, c=9$. The angle and the phase speed are well recovered while the wave number differs by a factor of 2. In Fig. 12, we show the theoretical data from the Mercier and Normand predictions, Smith and Davis prediction, which does not depend on $h$, and the experimental data. Qualitatively, a similar behavior is found between the MN values and the experimental ones when $h$ is varied. Nevertheless, a quantitative comparison fails, mainly because of the wave number values, which are always smaller (typically one half) in the experiment. Two reasons can explain this difference. The first one is the finite size of the experimental cell because it can influence the basic flow even far from the cell walls. This effect is not considered in the numerical analysis. The second one is the discrepancy found between the experimental and numerical temperature $z$-profiles below the threshold (Fig. 5 and MN).

\section{Nonlinear behavior}

Smith performed a nonlinear stability analysis ${ }^{11}$ of the SD solutions. He predicted the traveling wave (resp. mixed 
wave) solution to be stable (resp. unstable) for $P=10$. We confirm this prediction for $h>h_{c}$, reporting a single inclined traveling-wave. However, for $h<h_{c}$, the shape of the source deserves a two-dimensional description ${ }^{41}$ which has not been made as far as we know.

\section{Stationary rolls}

Stationary patterns for this buoyant-thermocapillary problem have been predicted by Mercier and Normand. ${ }^{9}$ According to Fig. 1(b) of MN, two unstably stratified layers can develop near the surface and the bottom plate for positive $B i$. The above layer destabilizes against Bénard-Marangoni instability whereas the bottom layer can be Rayleigh-Bénard unstable. This calculation, made for a bottom plate with a fixed linear temperature gradient, predicts the bottom layer to be thicker and to transit first.

In our system, the vertical temperature profile is unstable only in the upper layer (Fig. 5). This difference can be due to the finite conductivity of the glass bottom plate and thus to possible heat flux across.

\section{Comparison with experimental results in large rectangular channels}

Experiments in large rectangular containers with similar horizontal gradient have been performed by Riley and Neitzel $^{27}$ (RN) and by Pelacho and Burguete ${ }^{28}$ (PB). Both experiments use $L_{y}=50 \mathrm{~mm}$, a much smaller value than ours. Onset values have been plotted in Fig. 20 together with our results for $L_{x}=30 \mathrm{~mm}$ and various $L_{y}$. The data for $L_{x}$ $=10$ and $20 \mathrm{~mm}$ are not represented (cf. Sec. IV A 1). All data exhibit the same evolution with Bond number. The only discrepancy concerns the lowest value of $B o$ (small $h$ ) for our data in Table III. This discrepancy has been noted by RN and is explained by the fact that the waves solution at threshold are strongly inhomogeneous in our long cell. This is reinforced by our choice to report onset only when the whole cell is invaded, in order to compare with the uniform theoretical solution. Complementary studies have considered shorter $L_{y}=90 \mathrm{~mm}$ and $L_{y}=180 \mathrm{~mm}$ cells where the first apparition of waves is reported as onset. Onsets are much lower for small $h$ and in perfect agreement with $\mathrm{RN}$ and $\mathrm{PB}$ data (Fig. 20). Let us note that the main characteristics of the waves are close to RN and PB data. We also found comparable values for the effective gradient near oscillation threshold for RN data $(0.75<h<1.75)$. Riley and Neitzel present two oscillating modes: Hydrothermal waves (HTW) for $h$ $<1.3 \mathrm{~mm}$ and oscillarory multicellular cells (OMC) above. In our longer cell, we clearly observe the hydrothermal waves to superimpose on steady corotating rolls along $y$ axis. ${ }^{37}$ We believe that RN's oscillatory multicellular cells also represent this combination.

\section{SUMMARY AND CONCLUSION}

Experimental results obtained in a lateral heating experiment have been presented. Varying the experimental parameters $h, L_{x}, L_{y}$, and $\Delta T$, various stable states have been characterized. The basic flow that appears as soon as $\Delta T$ is not null, can be destabilized producing hydrothermal waves (for $\left.h<h_{r}\right)$ or stationary rolls $\left(h>h_{r}\right)$.

The observed hydrothermal waves correspond to bulk temperature oscillations, and exhibit different behaviors depending on the value of $h$. For $h<h_{c}$, the surface stresses are dominant, and the characteristics of the planar waves are very close to those predicted by Smith and Davis. ${ }^{3}$ For $h$ $>h_{c}$, their description is no more applicable because of the vertical temperature gradients that appear across the fluid layer, amplifying buoyancy effects. For these values of $h$, the predicted results of Mercier and Normand, ${ }^{9}$ including buoyant forces, are recovered except the wave number value. The quantitative discrepancy could be due to finite size considerations that alter the vertical velocity and temperature profiles. The spatial structure of the wave pattern reveals two types of wave-sources depending on the fluid depth. We believe these sources to trace back the existence of two different types of waves: one-dimensional waves for $h>h_{c}$ and twodimensional waves for $h<h_{c} \cdot{ }^{37,40}$ For large $h$, when buoyant forces dominate, the basic flow destabilizes in the form of a stationary pattern, which can be related to BénardMarangoni convection.

This set of experiments brought many quantitative data varying most control parameters of the system. In trying to reduce the set of relevant parameters, we emphasize the role of $h^{3} / L_{x}$ as the spatial scale of the problem. This parameter appears in a natural way when the Bond number is expressed using a Marangoni number based on horizontal scale $L_{x}$ and a Rayleigh number based on vertical scale $h$.

\section{ACKNOWLEDGMENTS}

The authors thank M. A. Pelacho, A. Garcimartín, H. Mancini, C. Pérez-García, J. F. Mercier, and C. Normand for stimulating discussions and C. Gasquet, M. Labouise, and B. Ozenda for their technical assistance. We also thank J. K. Platten for the measurement of the oil surface tension. J.B. acknowledges a postdoctoral grant from SEUID (Spanish Government) and financial support through contracts PB980208 (Spanish Government) and PIUNA (Universidad de Navarra, Spain).

\footnotetext{
${ }^{1}$ F. Preisser, D. Schwabe, and A. Scharmann, "Steady and oscillatory thermocapillary convection in liquid columns with free cylindrical surface," J. Fluid Mech. 126, 545 (1983).

${ }^{2}$ M. C. Cross and P. C. Hohenberg, "Pattern formation outside of equilibrium," Rev. Mod. Phys. 65, 851 (1993).

${ }^{3}$ M. K. Smith and S. H. Davis, "Instabilities of dynamic thermocapillary liquid layers. Part 1. Convective instabilities," J. Fluid Mech. 132, 119 (1983).

${ }^{4}$ S. H. Davis, "Thermocapillary instabilities," Annu. Rev. Fluid Mech. 19, 403 (1987).

${ }^{5}$ M. K. Smith, "Instability mechanisms in dynamic thermocapillary liquid layers," Phys. Fluids 29, 3182 (1986).

${ }^{6}$ P. Laure and B. Roux, "Linear and non-linear analysis of the Hadley circulation," J. Cryst. Growth 97, 226 (1989).

${ }^{7}$ G. Z. Gershuni, P. Laure, V. M. Myznikov, B. Roux, and E. M. Zhukhovitsky, "On the stability of plane-parallel advective flows in long horizontal layers," Microgravity Q. 2, 141 (1992).

${ }^{8}$ P. Parmentier, V. Regnier, and G. Lebon, "Buoyant-thermocapillary insta-
} 
bilities in medium-Prandtl number fluid layers subject to a horizontal temperature gradient," Int. J. Heat Mass Transf. 36, 2417 (1993).

${ }^{9}$ J. F. Mercier and C. Normand, "Buoyant-thermocapillary instabilities of differentially heated liquid layers," Phys. Fluids 8, 1433 (1996).

${ }^{10}$ J. Priede and G. Gerbeth, "Convective, absolute and global instabilities of thermocapillary-buoyancy convection in extended layers," Phys. Rev. E 56, 4187 (1997).

${ }^{11}$ M. K. Smith, "The nonlinear stability of dynamic thermocapillary liquid layers," J. Fluid Mech. 194, 391 (1988).

${ }^{12}$ B. M. Carpenter and G. M. Homsy, "Combined buoyant-thermocapillary flow in a cavity," J. Fluid Mech. 207, 121 (1989).

${ }^{13} \mathrm{H}$. Ben Hadid and B. Roux, "Buoyancy and thermocapillary-driven flows in differentially heated cavities for low Prandtl number fluids," J. Fluid Mech. 235, 1 (1992).

${ }^{14}$ D. Villers and J. K. Platten, "Coupled buoyancy and Marangoni convection in acetone: experiments and comparison with numerical simulations," J. Fluid Mech. 234, 487 (1992).

${ }^{15}$ J. F. Mercier and C. Normand, "Influence of the Prandtl number on the location of recirculation eddies in thermocapillary flows," Int. J. Heat Mass Transf. (in preparation).

${ }^{16}$ M. Mundrane and A. Zebib, "Oscillatory buoyant thermocapillary flow," Phys. Fluids 6, 3294 (1994).

${ }^{17} \mathrm{~J}$. Xu and A. Zebib, "Oscillatory two- and three-dimensional thermocapillary convection,” J. Fluid Mech. 364, 187 (1998).

${ }^{18}$ R. Velten, D. Schwabe, and A. Scharmann, "The periodic instability of thermocapillary convection in cylindrical liquid bridges," Phys. Fluids A 3, 267 (1991).

${ }^{19}$ C. De Saedeleer, A. Garcimartín, G. Chavepeyer, J. K. Platten, and G. Lebon, "The instability of a liquid layer heated from the side when the upper surface is open to air," Phys. Fluids 8, 670 (1996).

${ }^{20}$ A. Garcimartín, N. Mukolobwiez, and F. Daviaud, "Origin of surface waves in surface tension driven convection," Phys. Rev. E 56, 1699 (1997).

${ }^{21}$ A. B. Ezersky, A. Garcimartín, J. Burguete, H. L. Mancini, and C. PerezGarcia, "Hydrothermal waves in Marangoni convection in a cylindrical container," Phys. Rev. E 47, 1126 (1993).

${ }^{22}$ A. B. Ezersky, A. Garcimartín, H. L. Mancini, and C. Perez-Garcia, "Spatiotemporal structure of hydrothermal waves in Marangoni convection," Phys. Rev. E 48, 4414 (1993)

${ }^{23}$ D. Schwabe, U. Möller, J. Schneider, and A. Scharmann, "Instabilities of shallow thermocapillary liquid layers," Phys. Fluids A 4, 2368 (1992).

${ }^{24}$ J. Schneider, D. Schwabe, and A. Scharmann, "Experiments on surface waves in dynamic thermocapillary liquid layers," Microgravity Sci. Technol. 9, 86 (1996)

${ }^{25}$ M. K. Smith and S. H. Davis, "Instabilities of dynamic thermocapillary liquid layers. Part 2. Surface-waves instabilities," J. Fluid Mech. 132, 145 (1983).

${ }^{26} \mathrm{~F}$. Daviaud and J. M. Vince, "Traveling waves in a fluid layer subjected to a horizontal temperature gradient," Phys. Rev. E 48, 4432 (1993).

${ }^{27}$ R. J. Riley and G. P. Neitzel, "Instability of thermocapillary-buoyancy convection in shallow layers. Part 1. Characterization of steady and oscillatory instabilities," J. Fluid Mech. 359, 143 (1998).

${ }^{28}$ M. A. Pelacho and J. Burguete, "Temperature oscillations of hydrothermal waves in thermocapillary-buoyancy convection," Phys. Rev. E 59, 835 (1999).

${ }^{29}$ Y. Kamotani, J. Masud, and A. Pline, "Oscillatory convection due to combined buoyancy and thermocapillarity," J. Thermophys. Heat Trans. 10, 102 (1996).

${ }^{30}$ P. Gillon and G. M. Homsy, "Combined thermocapillary-buoyancy convection in a cavity: An experimental study," Phys. Fluids 8, 2953 (1996).

${ }^{31}$ M. G. Braunsfurth and G. M. Homsy, "Combined thermocapillarybuoyancy convection in a cavity. Part II. An experimental study," Phys. Fluids 9, 1277 (1997).

${ }^{32}$ N. Mukolobwiez, A. Chiffaudel, and F. Daviaud, "Supercritical Eckhaus instability for surface-tension-driven hydrothermal waves," Phys. Rev. Lett. 80, 4661 (1998).

${ }^{33}$ J. Burguete, H. Chaté, F. Daviaud, and N. Mukolobwiez, "Hydrothermal wave amplitude holes in a lateral heating convection experiment," Phys. Rev. Lett. 82, 3252 (1999).

${ }^{34}$ J. M. Vince and M. Dubois, "Hot wire below the free surface of a liquid: structural and dynamical properties of a secondary instability," Europhys. Lett. 20, 505 (1992).

${ }^{35}$ E. Favre, L. Blumenfeld, and F. Daviaud, "Instabilities of a liquid layer locally heated on its free surface," Phys. Fluids 9, 1473 (1997).

${ }^{36} \mathrm{~N}$. Mukolobwiez, "Etudes de systemes d'ondes propagatives dans de ecoulements thermogravitaires et thermocapillaires," Ph.D. thesis, Université Paris XI, France, 1998.

${ }^{37} \mathrm{~N}$. Garnier, "Ondes non lineaires a une et deux dimensions dans une mince couche de fluide," Ph.D. thesis, Université Paris 7, France, 2000.

${ }^{38}$ D. Schwabe and H. Dürr, "Holographic interferometry and flow visualization by tracers applied to buoyant-thermocapillary convection in an open rectangular gap," Microgravity Sci. Technol. 9, 201 (1996).

${ }^{39}$ N. Garnier and A. Chiffaudel, "Non-linear transition to a global mode for traveling-wave instability in a finite box," Phys. Rev. Lett. 86, 75 (2001).

${ }^{40}$ N. Garnier and A. Chiffaudel, "Two dimensional hydrothermal waves in an extended cylindrical vessel," Eur. Phys. J. B 19, 87 (2001).

${ }^{41}$ N. Garnier, A. Chiffaudel, and F. Daviaud, "Transition 1D-2D pour des ondes hydrothermales non-linéaires," in 2ème Rencontre du Non-linéaire IHP Paris 1999, edited by Y. Pomeau and R. Ribotta (Paris Onze Editions, Orsay, 1999), pp. 193-198 (in French).

${ }^{42}$ M. A. Pelacho, A. Garcimartín, and J. Burguete, "Local Marangoni number at the onset of hydrothermal waves," Phys. Rev. E 62, 477 (2000).

${ }^{43}$ J. R. A. Pearson, "On convection cells induced by surface tension," J. Fluid Mech. 4, 489 (1958).

${ }^{44}$ M. Dubois, R. Da Silva, F. Daviaud, P. Bergé, and A. Petrov, "Collective oscillating mode in a 1D chain of convective rolls," Europhys. Lett. 8, 135 (1989).

${ }^{45} \mathrm{~J}$. Burguete, "Inestabilidades producidas por un calentamiento localizado," Ph.D. thesis, Universidad de Navarra, Spain, 1995. 